\title{
An End-to-End Error Model for Classification Methods Based on Temporal Change or Polarization Ratio of SAR Intensities
}

\author{
Alexandre Bouvet, Member, IEEE, Thuy Le Toan, Nicolas Floury, Member, IEEE, and Trevor Macklin
}

\begin{abstract}
This paper aims at defining the expression of the probability of error of classification methods using a synthetic aperture radar (SAR) intensity ratio as a classification feature. The two SAR intensities involved in this ratio can be measurements from different dates, polarizations, or, also possibly, frequency bands. Previous works provided a baseline expression of the probability of error addressing the two-class problem with equal $a$ priori class probabilities and no calibration error. This study brings up a novel expression of the error, providing the possibility to assess the effect of class probabilities and calibration errors. An extended expression is described for the $n$-class problem. The effect of calibration errors such as channel gain imbalance, radiometric stability, and crosstalk is assessed in the general case. The results indicate that, for the applications under study, channel gain imbalance is usually not a decisive parameter, but radiometric stability is more critical in methods based on the temporal change. Crosstalk has a negligible effect in the case of copolarizations. The impacts of other system parameters, such as ambiguity ratio, time-lapse between repeat-pass orbits, spatial resolution, and number of looks, are illustrated through a set of assumptions on the backscattering values of the considered classes. The model is validated by comparing some of its outputs to experimental results calculated from the application of rice fields mapping methods on real data. This error model constitutes a tool for the design of future SAR missions and for the development of robust classification methods using existing SAR instruments.
\end{abstract}

Index Terms-Calibration, image classification, polarization ratio, synthetic aperture radar, temporal change.

\section{INTRODUCTION}

$\mathbf{I}$ N THE PAST few years, several satellites have been launched with a fully polarimetric synthetic aperture radar (SAR) onboard. Polarimetric data contain coherent acquisitions of the four polarizations and thus carry much more information

Manuscript received April 30, 2009; revised February 3, 2010. Date of publication June 14, 2010; date of current version August 25, 2010. The ENVISAT/ASAR data used in Section V were provided by the European Space Agency (Cat-1 AO project 697).

A. Bouvet was with the Centre d'Etudes Spatiales de la Biosphère, 31401 Toulouse, France. He is now with the European Commission Joint Research Center, 21020 Ispra, Italy (e-mail: alexandre.bouvet@jrc.it).

T. Le Toan is with the Centre d'Etudes Spatiales de la Biosphère, 31401 Toulouse, France.

N. Floury is with the European Space Agency, European Space Research and Technology Centre (ESA-ESTEC), 2200 AG Noordwijk, The Netherlands.

T. Macklin is with the Networks, Security and Information Systems Department, BAE SYSTEMS Advanced Technology Centre, CM2 8HN Chelmsford Essex, U.K

Color versions of one or more of the figures in this paper are available online at http://ieeexplore.ieee.org.

Digital Object Identifier 10.1109/TGRS.2010.2047399 than single-polarized or incoherently dual-polarized data. To exploit this information, polarimetric classification methods have been developed in the end of the 1990s [1]-[5] and have been applied to airborne polarimetric SAR data with excellent accuracies. Nonetheless, because of constraints related to the pulse repetition frequency (PRF), to the data rate, and to the high spatial resolution required to enhance polarimetric features, the classification performance in these coherent systems is usually traded against a much smaller swath when compared to a similar incoherent acquisition (single or dual polarization). Effectively, the swath widths of the polarimetric imagery data in TerraSAR-X, Radarsat-2, and PALSAR are 15, 25, and $30 \mathrm{~km}$, respectively, while some single-polarization SARs provide data with a swath as wide as $500 \mathrm{~km}$, with a coarser spatial resolution though. Some remote sensing applications involve mapping of large areas or frequent observations and therefore require a large spatial coverage (and consequently a high revisit frequency) rather than a high spatial resolution. For this reason, the future SAR planned for launch by ESA in 2011 (Sentinel-1), which aims at providing SAR data operationally with a high temporal resolution, will be incoherently dual polarized. Moreover, all the actual SARs including a fully polarimetric mode are also able to provide single- or dual-polarization data at larger swaths than their fully polarimetric data, and the 18-year satellite SAR archive data contain only incoherent imagery data sets. For all these reasons, classification methods that are specific to incoherent SAR data are still needed.

A number of easy-to-implement classification methods using incoherent SAR data are based on the ratio of two intensity (backscattering coefficient) images, used as classification feature. For example, a widely used feature in single-channel SAR data (one frequency and one polarization) is the temporal change (TC) of the intensity between two dates. This classification feature is derived from the ratio of the backscattering coefficient images at two dates, rather than from the difference of the backscattering coefficients. Indeed, the latter was shown to produce larger errors in high-intensity regions than in low-intensity regions [6]. The temporal intensity ratio method has been widely used since satellite SAR systems have been available in the early 1990s (ERS-1 and RADARSAT-1 at C-band and JERS at L-band), which provided data periodically. In recent years, multipolarization systems, such as ASAR onboard ENVISAT (dual polarization and C-band), PALSAR onboard ALOS (dual and quad polarizations and L-band), RADARSAT-2 (dual and quad polarizations and C-band), and 
TerraSAR-X (dual and quad polarizations and X-band), have become available, making it possible to use classifiers based on the polarization ratio (PR) at a single date, i.e., the ratio of two backscattering images at the same date at two different polarizations.

Applications of the TC of SAR intensity between two dates in classification methods include the detection of events such as floods with JERS [7] and ASAR [8], deforestation with ERS-1 and JERS [9] or harrowing in fields using ASAR [10], and the mapping of rice fields with ERS-1 [11] and Radarsat-1 [12]. Classification features based on PR have been extensively demonstrated in a wide range of applications: oil slick detection with $\mathrm{Ka}$ - and C-band $\mathrm{HH} / \mathrm{VV}$ [13]; discrimination of vegetated fields from bare soil with C-band HV/HH and HV/VV [14]; discrimination of broad-leaf crops from small-stem crops with C-band RR/RL [14], where R and L denote right and left circular polarizations; crop classification with $\mathrm{C}$ - or L-band $\mathrm{HH} / \mathrm{HV}$ [15]; rice or wheat fields mapping using C-band HH/VV [16], [17]; and discrimination of multiyear sea ice from first-year sea ice using C-band $\mathrm{HV} / \mathrm{HH}$ [18].

The accuracy of such classification methods based on an intensity ratio has been assessed in [6] for the two-class problem, taking into account the target characteristics. However, the impacts of the system parameters on the classification performance were not addressed. Those parameters include spatial resolution, ambiguity, orbit repeat cycle, channel gain imbalance, radiometric stability, and crosstalk. For the assessment of the classification robustness and for the design of future SAR missions, there is a need to extend the study in [6] by considering the system parameters in the assessment of the classification performance.

The objective of this paper is to provide the general formulation of the error in classification methods based on a SAR intensity ratio, in such a way that the impact of the system parameters can be assessed. In Section II, we calculate the theoretical probability of error of such methods for a two-class problem, with an extension scheme to the $n$-class problem. In Section III, the impact of calibration parameters (radiometric accuracy, radiometric stability, channel gain imbalance, and crosstalk) on the probability of error is calculated for the general case. Section IV addresses the effect of other system and processing parameters, such as ambiguity ratio, time-lapse between repeat-pass orbits, number of looks, and spatial resolution, under a set of assumptions on the backscattering profiles of the classes. The model is validated experimentally in Section V by applying rice fields mapping methods to real SAR data.

\section{THEORETICAL EXPRESSION OF THE PROBABILITY OF ERROR}

\section{A. Description of the Classification Algorithm}

We want to develop a classification method using a SAR intensity ratio $r=I_{2} / I_{1}$ as a classification feature. The backscatter intensity $I$ can represent any of the following backscattering coefficients: $\sigma^{0}, \beta^{0}$, or $\gamma$. For a homogeneous area in a SAR image with a number of looks equal to $L$, the backscattering intensity $I$ can be modeled as a gamma distribution with the shape parameter equal to $L$ and the scale parameter equal to $\langle I\rangle / L$, where $\langle\cdot\rangle$ denotes the average value over a homogeneous area [19]. Its probability density function (pdf) is thus equal to

$$
p(I \mid\langle I\rangle)=\frac{L^{L} I^{L-1}}{\langle I\rangle^{L} \Gamma(L)} \exp \left(-\frac{L I}{\langle I\rangle}\right)
$$

where $\Gamma(L)$ represents the Gamma function $\Gamma(x)=$ $\int_{0}^{\infty} t^{x-1} e^{-t} d t$.

When $I_{1}$ and $I_{2}$ come from uncorrelated channels, the pdf of the intensity ratio $r=I_{2} / I_{1}$ of a homogeneous region is found to depend only on the ratio of average intensities $\bar{r}=\left\langle I_{2}\right\rangle /\left\langle I_{1}\right\rangle$ and not specifically on the average intensities $\left\langle I_{1}\right\rangle$ and $\left\langle I_{2}\right\rangle[19]$

$$
p\left(r \mid\left\langle I_{1}\right\rangle,\left\langle I_{2}\right\rangle\right)=\frac{\Gamma(2 L)}{\Gamma(L)^{2}} \frac{\bar{r}^{L} r^{L-1}}{(\bar{r}+r)^{2 L}} .
$$

In the methods based on $\mathrm{TC}$, the condition of uncorrelated channels is well met when dealing with agricultural areas at $\mathrm{X}$ - or C-band, because changes occur between two repeat-pass data. For the PR method, the correlation between channels is low when the two polarizations involve different backscattering mechanisms. This is the case for $\mathrm{HH}$ and VV at $\mathrm{X}$ - or C-band on crops with a vertical structure (rice and wheat) or for $\mathrm{HH}$ and $\mathrm{HV}$ on agricultural areas at X- or C-band and on forests at L- or P-band. In these common examples, the two channels forming the intensity ratio are not strongly correlated. We will first assume in our analysis that the two channels are uncorrelated. The correlated case will be investigated at the end of this section.

We consider two classes $\mathrm{A}$ and $\mathrm{B}$, characterized by mean intensity ratios $r_{A}=\left\langle I_{2, A}\right\rangle /\left\langle I_{1, A}\right\rangle$ and $r_{B}=\left\langle I_{2, B}\right\rangle /\left\langle I_{1, B}\right\rangle$, supposing that $r_{B}>r_{A}$. We adopt here a Bayesian approach to decide on whether to classify a pixel having a given intensity ratio $r$ into class $\mathrm{A}$ or $\mathrm{B}$. Bayes' theorem states

$$
p(A \mid r)=\frac{p(r \mid A)}{p(r)} p(A)
$$

where $p(A \mid r)$ represents the probability for a pixel with an intensity ratio $r$ to belong to class $\mathrm{A} ; p(r \mid A)$ represents the probability for a pixel belonging to class A to have an intensity ratio that is equal to $r$, which is given in (2) with $\bar{r}=r_{A} ; p(A)$ represents the a priori probability of class $\mathrm{A}$ in the scene; and $p(r)$ represents the probability for the intensity ratio to be equal to $r$ in the image. The same relationship applies for class B.

The classification algorithm consists in assigning a pixel with an intensity ratio $r$ to class $\mathrm{B}$ whenever $p(B \mid r)>p(A \mid r)$. This inequality is rewritten using (2) and (3)

$$
\frac{\Gamma(2 L)}{\Gamma(L)^{2}} \frac{r_{B}^{L} r^{L-1}}{\left(r_{B}+r\right)^{2 L}} \frac{p(B)}{p(r)}>\frac{\Gamma(2 L)}{\Gamma(L)^{2}} \frac{r_{A}^{L} r^{L-1}}{\left(r_{A}+r\right)^{2 L}} \frac{p(A)}{p(r)}
$$

and it leads eventually to

$$
r>\sqrt{r_{A} r_{B}} \cdot \frac{\sqrt{\frac{r_{B}}{r_{A}}}\left(\frac{p(A)}{p(B)}\right)^{\frac{1}{2 L}}-1}{\sqrt{\frac{r_{B}}{r_{A}}}-\left(\frac{p(A)}{p(B)}\right)^{\frac{1}{2 L}}}=r_{\mathrm{opt}} .
$$

The Bayesian approach therefore reduces to simply thresholding the intensity ratio using an optimal classification threshold 
$r_{\text {opt }}$ given in (4). This threshold depends on the following four parameters: $r_{A}, r_{B}, p(B)$ or $p(A)$, and $L$. In the case of prior equiprobability, i.e., when $p(A)=p(B)=0.5$, the optimal classification threshold takes a particular value, noted as $r_{0}$

$$
r_{0}=\sqrt{r_{A} r_{B}}
$$

It is also to be noted that, when the number of looks $L$ increases, the dependence of the optimal threshold on the a priori class probabilities strongly decreases due to the $1 / 2 L$ power. With a high number of looks (for example $L>32$ ), we can therefore consider that $r_{\mathrm{opt}} \approx r_{0}$.

\section{B. Calculation of the Probability of Error}

Rignot and van Zyl [6] calculated the probability of error in a classification method using a threshold on the ratio of backscatter intensities at two dates $r_{\mathrm{TC}}=I_{p, d 2} / I_{p, d 1}$, where $p$ is the polarization and $d 1$ and $d 2$ are the dates, in the case of equiprobable classes. The approach is valid for any other backscatter intensity ratio, including PRs $r_{\mathrm{PR}}=I_{p 2, d} / I_{p 1, d}$, where $p 1$ and $p 2$ are the polarizations and $d$ is the date. This section builds on this study to give an alternative formulation of the probability of error of the method, involving a supplementary parameter, and valid for any a priori class probabilities.

We use a threshold $r_{t}$ to classify a pixel with intensity ratio $r$ in to class A or B. Ideally, $r_{t}$ is equal to the optimal threshold $r_{\text {opt }}$ when the latter is known or can be calculated, but we consider here the general case valid for any value of $r_{t}$.

The probability of error for class $\mathrm{A}$, noted as $P E_{A}$, corresponding to the probability of classifying a pixel belonging to class A into class B, and the probability of error for class B, noted as $P E_{B}$, are then given by

$$
\begin{aligned}
P E_{A} & =\int_{r_{t}}^{\infty} p\left(r \mid r_{A}\right) d r \\
P E_{B} & =\int_{0}^{r_{t}} p\left(r \mid r_{B}\right) d r .
\end{aligned}
$$

The total probability of error is thus

$$
P E=p(A) \cdot P E_{A}+p(B) \cdot P E_{B} .
$$

Fig. 1 shows the pdf of two classes with $r_{A}=0 \mathrm{~dB}$ and $r_{B}=$ $6 \mathrm{~dB}$ for a number of looks that are equal to ten, together with their probability of error $P E_{A}$ and $P E_{B}$, when the classification threshold is equal to $r_{t}=2.5 \mathrm{~dB}$.

In order to simplify the calculations, the classification threshold $r_{t}$ is expressed relatively to the optimal threshold for equal a priori class probabilities $r_{0}$ through the use of a new parameter that is noted as $d$, so that $r_{t}=d \cdot r_{0}$. We also introduce parameter $\Delta r=r_{B} / r_{A}$, which represents the distance between the mean intensity ratios of the two classes, and it is, therefore, a measure of the class separability. This parameter is more conveniently expressed in decibels $(\Delta r)_{\mathrm{dB}}=\left(r_{B}\right)_{\mathrm{dB}}-\left(r_{A}\right)_{\mathrm{dB}}$.

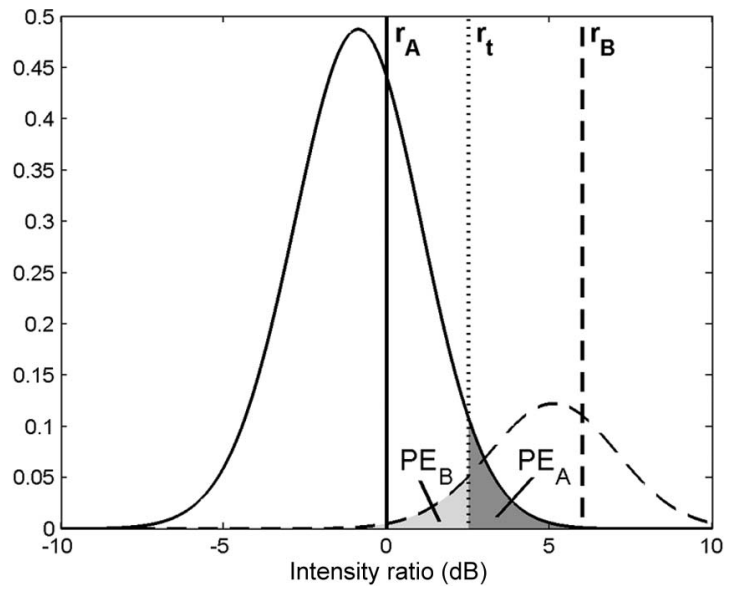

Fig. 1. PDFs of the intensity ratio of (full line) class A and (dashed line) class B with class parameters $r_{A}=0 \mathrm{~dB}$ and $r_{B}=6 \mathrm{~dB}$ for $L=10$. The class parameters are represented by vertical lines (full and dashed), and the chosen classification threshold $r_{t}$ is represented by a vertical dotted line. The colored areas represent the probability of error for each class (dark gray) $P E_{A}$ and (light gray) $P E_{B}$.

In the general case of unknown a priori probabilities, we find that the probability of error is (see Appendix I)

$$
P E=(1-p(B)) \cdot h_{L}\left(d^{2} \cdot \Delta r\right)+p(B) \cdot h_{L}\left(\frac{\Delta r}{d^{2}}\right)
$$

where

$$
h_{L}(\Delta r)=\frac{\Gamma(2 L)}{\Gamma(L)^{2}} \sum_{k=0}^{\infty}\left(\begin{array}{c}
L-1 \\
k
\end{array}\right) \frac{(-1)^{k}}{L+k} \frac{1}{(1+\sqrt{\Delta r})^{L+k}}
$$

with $\Delta r=r_{B} / r_{A}$ and $\left(\begin{array}{c}L-1 \\ k\end{array}\right)=\Gamma(L) /(\Gamma(k+1) \Gamma(L-k))$, representing the binomial coefficient.

It is to be noted that, when $L$ is an integer, $\left(\begin{array}{c}L-1 \\ k\end{array}\right)$ is equal to zero for $k$ greater than or equal to $L$, and $h_{L}$ is thus easier to compute

$$
h_{L}(\Delta r)=\frac{\Gamma(2 L)}{\Gamma(L)^{2}} \times \sum_{k=0}^{L-1}\left(\begin{array}{c}
L-1 \\
k
\end{array}\right) \frac{(-1)^{k}}{(L+k)} \frac{1}{(1+\sqrt{\Delta r})^{L+k}} .
$$

The probability of error of the classification method therefore depends on the following four parameters:

1) $L$, which is the number of looks of the intensity images;

2) $\Delta r$, which is a measurement of the distance between the mean ratios of the two classes;

3) $p(B)$, which is the a priori probability of class $\mathbf{B}$;

4) $d$, which is a measurement of the distance between the retained classification threshold $r_{t}$ and the threshold $r_{0}$ (optimal threshold when the two classes are equiprobable).

1) Equal A Priori Probabilities: When $p(A)=p(B)$, the retained classification threshold $r_{t}$ is optimal when it is equal to $r_{0}$. Therefore, parameter $d=r_{t} / r_{0}$ is equal to one, and the expression of the probability of error reduces to

$$
P E=h_{L}(\Delta r) .
$$






Fig. 2. Probability of error (in percent) of the ratio method as a function of the change in intensity ratio $\Delta r$ (in decibels) between the two classes for a number of looks $L$ varying between 1 and 128 .

Fig. 2 shows the probability of error $P E$ as a function of $\Delta r$ for different values of $L$ when the classification threshold is $r_{0}$.

The figure can be used to assess the number of looks required to achieve a given accuracy in the classification when $r_{A}$ and $r_{B}$ are known.

Fig. 2 can also be used to assess the accuracy that can be expected when the system parameters (number of looks) and class characteristics $\left(r_{A}\right.$ and $\left.r_{B}\right)$ are known. Unsurprisingly, the error decreases when $L$ and $\Delta r$ increase.

2) General Case: We can derive from (4) that the optimal threshold is found for a specific value of parameter $d$

$$
d=d_{\mathrm{opt}}=r_{\mathrm{opt}} / r_{0}=\frac{\sqrt{\Delta r}\left(\frac{1-p(B)}{p(B)}\right)^{\frac{1}{2 L}}-1}{\sqrt{\Delta r}-\left(\frac{1-p(B)}{p(B)}\right)^{\frac{1}{2 L}}} .
$$

Thus, for the general case, the optimal classification threshold depends on $r_{0}, L, \Delta r$, and $p(B)$. However, when the method is to be used in several different scenes, $p(B)$ is not known in most cases. It is then suggested to use $r_{0}$ as a threshold in the classification scheme. The additional classification error compared to the optimal case needs to be assessed in order to make sure that such a practice is acceptable.

Fig. 3 shows the additional error due to the use of $r_{0}$ rather than $r_{\mathrm{opt}}$ as a function of the optimal probability of error $P E_{\mathrm{opt}}$ found for $r_{\mathrm{opt}}$, with a fixed number of looks $L$ equal to eight and $p(B)$ varying from 0.5 to 0.9 [Fig. 3(a)] and with a fixed $p(B)$ that is equal to 0.8 and a number of looks $L$ varying from 1 to 128 [Fig. 3(b)]. The variations of $P E_{\text {opt }}$ account for different values of $\Delta r$. The additional error for $p(B)=p_{B}$ is equal to that for $p(B)=1-p_{B}$, as it can be derived by combining (12) and (9). Therefore, Fig. 3 can be read for the values of $p(B)$ from 0.1 to 0.4 as well.

Fig. 3(a) shows that, for a fixed number of looks (here, $L=8$ ), the additional error increases when the class proportions differ from equiprobability, for any value of $P E_{\mathrm{opt}}$, i.e., for any value of $\Delta r$. However, among cases most likely to be encountered $(0.2 \leq p(B) \leq 0.8)$, those leading to an acceptable accuracy (i.e., $\Delta r$ values corresponding to $P E_{\text {opt }}$ lower than $15 \%)$ provide only slightly suboptimal results $(7 \%$ of the



(a)

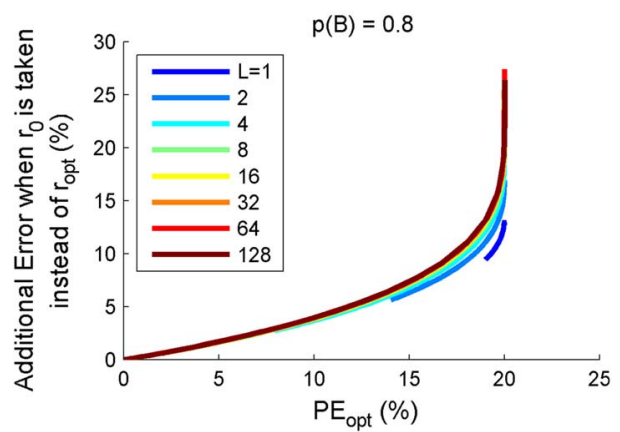

(b)

Fig. 3. Additional error due to the use of $r_{0}$ rather than $r_{\mathrm{opt}}$ as a function of the optimal $P E$ (a) for different values of $p(B)$ from 0.5 to 0.9 with $L=8$ and (b) for different values of $L$ from 1 to 128 with $p(B)=0.8$.

additional error in the worst case) when $r_{0}$ is used instead of the true optimal threshold $r_{\text {opt }}$. Fig. 3(b) shows that this is true whatever is the number of looks.

In this case, when the classification threshold is taken to be equal to $r_{0}$ instead of $r_{\mathrm{opt}}$, the probability of error is the same as that of the equal a priori probabilities case, which is given by (11), and Fig. 2 can be used as well.

Therefore, although it is a practical necessity, the use of $r_{0}$ instead of $r_{\mathrm{opt}}$ as a classification threshold has a limited negative impact on the classification accuracy in most cases $\left(0.2 \leq p(B) \leq 0.8, P E_{\mathrm{opt}}<15 \%\right)$, and in addition, it leads to an expression of the error that is independent of the a priori probabilities of the two classes.

In the rest of this paper, it is assumed that the classification threshold is $r_{0}$.

\section{Estimation of $r_{0}$}

In order to implement this classification method based on a SAR intensity ratio, the value of $r_{0}=\sqrt{r_{A} r_{B}}$ needs to be estimated, which requires the estimation of the mean intensity ratios of the two classes $r_{A}$ and $r_{B}$. The estimation of these two class parameters can be done in different ways. For example, supervised methods using either the maximum likelihood criterion or the histograms of the intensity ratios, such as the one described in [16], would be suitable. An unsupervised method has been presented in [20], and it can be used when the training data are not available. Alternatively, a prior-knowledge scheme can be adopted when $r_{A}$ and $r_{B}$ are known from previous studies involving intensity images that are similar to those used in the classification (same sensor or same image characteristics, 
namely, frequency, polarizations, and incidence angle) or from backscattering models. The impact of the system parameters, such as calibration imperfections, on the probability of error in the prior-knowledge procedure will therefore be higher than in the supervised or unsupervised methods.

\section{Extension to Multitemporal Data}

Two kinds of classification features based on an intensity ratio have been defined in Section II-A, where one is based on TC involving two dates $r_{\mathrm{TC}}=I_{p, d 2} / I_{p, d 1}$ and where the other corresponds to a PR at a single date $r_{\mathrm{PR}}=I_{p 2, d} / I_{p 1, d}$. When data sets containing more than two dates are available, multitemporal features should be defined for these two kinds of intensity ratios to improve the classification.

1) TC Method: Such classification methods are generally based on the assumption that the SAR intensity of one class remains relatively stable in time ( $r \approx 0 \mathrm{~dB}$ for any pair of date) while that of the other class would change.

If the expected change for this class is an increase in backscattering intensity that spans over a period longer than the satellite repeat cycle (for example, a plant growing season), the classification accuracy should be improved by considering a classification feature $r_{\mathrm{TC}, \text { multi }}=\max _{i, j>i}\left[I_{p, d j} / I_{p, d i}\right]$, which would maximize $r_{B}$ and leave $r_{A}$ nearly unchanged, with class B being the "changing" class and class A being the "stable" one. In that case, the probability of error $P E$ is hard to assess theoretically, but the multitemporal feature tends to increase the $\Delta r$ parameter and, thus, the accuracy.

Reversely, if the expected change is a decrease of value, then the classification feature should be $r_{\mathrm{TC} \text {,multi }}=$ $\max _{i, j>i}\left[I_{p, d i} / I_{p, d j}\right]$. If the relevant parameter is change in general (increase or decrease), the feature should be optimal for $r_{\mathrm{TC}, \text { multi }}=\max _{i, j>i}\left[\max \left(\left(I_{p, d j} / I_{p, d i}\right),\left(I_{p, d i} / I_{p, d j}\right)\right)\right]$. A double-threshold approach can also be adopted to account for increase and decrease, which would correspond to a three-class problem.

Otherwise, if the expected change occurs at a frequency higher than the repeat-pass frequency (such as changes due to the effect of weather), a more relevant parameter would be the mean change between two dates of the temporal series, which is similar to the mean annual variation introduced for the mapping of forested areas [21]

$$
r_{\mathrm{TC}, \text { multi }}=\frac{2}{N(N-1)} \sum_{i=1}^{N-1} \sum_{j=i+1}^{N} \max \left(\frac{I_{p, d j}}{I_{p, d i}}, \frac{I_{p, d i}}{I_{p, d j}}\right)
$$

where $N$ is the number of images in the multitemporal data set.

2) PR Method: In the case of a classification method based on a PR, the polarizations are generally chosen so that one of the classes exhibits large ratio values at least at some periods, while the other class remains relatively constant at lower values.

Therefore, the classification feature $r_{\mathrm{PR}, \text { multi }}=$ $\max _{i}\left[I_{p 2, d i} / I_{p 1, d i}\right]$ should be used to improve the classification accuracy by catching the optimal (highest) value of $r_{B}$ in the time series and keeping $r_{A}$ to low values.

\section{E. n-Class Problem}

As shown in (11), the two-class problem with equal a priori class probabilities and the use of $r_{0}$ as a classification threshold lead to an expression of the error $P E$ that is dependent on two parameters, namely, one being related to the SAR data characteristics (the number of looks $L$ ) and the other being related to the class characteristics (the distance between the mean intensity ratio of the two classes $\Delta r): P E=h_{L}(\Delta r)=$ $\operatorname{PE}(\Delta r, L)$.

A more general expression must be brought up in order to deal with cases when more classes are taken into account in the classification. Let us assume that $n$ classes are considered, with class $i$ characterized by a mean ratio $r_{i}$, and $r_{1}<r_{2}<$ $\cdots<r_{n}$. The distance between two consecutive mean ratios is $\Delta r_{i}=r_{i+1}-r_{i}$ (when expressed in decibels), with $i$ varying from $i$ to $n-1$. It can be shown that, for equiprobable classes, the overall classification error is (see Appendix II)

$$
P E=\frac{2}{n} \sum_{i=1}^{n-1} P E\left(\Delta r_{i}, L\right)=\frac{2(n-1)}{n} \operatorname{mean}_{i}\left[P E\left(\Delta r_{i}, L\right)\right] .
$$

For example, if all $\Delta r_{i}$ s are equal, this implies that the error of the $n$-class problem is increased by a factor $k_{n}=2(n-1) / n$ compared to the two-class problem $\left(k_{n}=1.33\right.$ for $n=3$, it is equal to 1.5 for $n=4$, and it tends to reach 2 for high values of $n$ ). For that reason, the classification method should be limited to few classes, all the more so as dealing with more classes will make them less likely to have high values of $\Delta r$.

\section{F. Case of Correlated Channels}

When the two channels are correlated, with a correlation coefficient $\rho$, their joint distribution can be modeled by Kibble's bivariate gamma distribution [22], and the pdf of the ratio is [23]

$$
p\left(r \mid\left\langle I_{1}\right\rangle,\left\langle I_{2}\right\rangle, \rho\right)=\frac{\Gamma(2 L)}{\Gamma(L)^{2}} \frac{\left(1-|\rho|^{2}\right)^{L} \bar{r}^{L}(r+\bar{r}) r^{L-1}}{\left((\bar{r}+r)^{2}-4|\rho|^{2} \bar{r} r\right)^{L+\frac{1}{2}}} .
$$

Fig. 4 shows the pdf of two classes with $r_{A}=0 \mathrm{~dB}$ and $r_{B}=6 \mathrm{~dB}$ for a number of looks equal to ten and for correlation coefficients $\rho$ ranging from 0 to 0.9 [Fig 4(a)], with a close-up on the region where the curves meet and where $P E$ is visualized [Fig 4(b)].

The optimal threshold is hard to express analytically using (6), but Fig. 4(b) shows that the crossing of the pdf of the two classes occurs at $r=r_{0}$ for any value of $\rho$, leading to the same optimal threshold under equal a priori class probabilities as for the case of uncorrelated channels.

Likewise, the probability of error is too complicated to be expressed in a satisfyingly explicit form, but it can be found from Fig. 4(b) that the area corresponding to $P E$ decreases when $\rho$ increases. The probability of error of classification methods based on a ratio of two correlated intensity channels is, therefore, lower than that corresponding to the case when the channels are uncorrelated, which is shown in Fig. 2. 


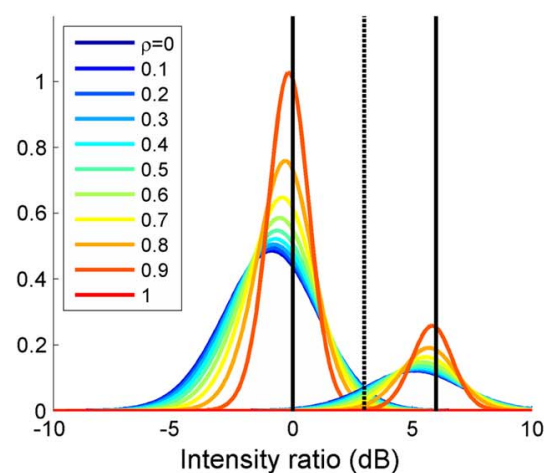

(a)



(b)

Fig. 4. (a) PDFs of classes A and B with class parameters $r_{A}=0 \mathrm{~dB}$ and $r_{B}=6 \mathrm{~dB}$ for $L=10$ and for correlation coefficients $\rho$ between channels ranging from 0 to 0.9 . The class parameters are represented by vertical full lines, and the classification threshold $r_{0}$ is represented by a vertical dashed line. (b) A close-up on the area where the curves meet is shown.

\section{IMPACT OF SAR CALIBRATION PARAMETERS}

When applying classification algorithms to SAR images, one must bear in mind the various imperfections that may affect the image quality of SAR products and must take them into account while evaluating the performance of such algorithms. We specifically address here the sensitivity of the classification methods to radiometric and polarimetric calibration imperfections. Calibration is characterized by a number of parameters, including radiometric accuracy and radiometric stability for the radiometric calibration, and crosstalk and channel gain imbalance for the polarimetric calibration.

Let $S$ represent the scattering matrix that characterizes the backscattering properties of the target

$$
S=\left(\begin{array}{cc}
S_{\mathrm{hh}} & S_{\mathrm{hv}} \\
S_{\mathrm{vh}} & S_{\mathrm{vv}}
\end{array}\right) .
$$

When taking into account the effects of the transmitting and receiving systems, the observed scattering matrix $Y$ that can be acquired by the radar system is [24]

$$
\left(\begin{array}{cc}
Y_{\mathrm{hh}} & Y_{\mathrm{hv}} \\
Y_{\mathrm{vh}} & Y_{\mathrm{vv}}
\end{array}\right)=A e^{j \Phi}\left(\begin{array}{cc}
1 & \delta_{2} \\
\delta_{1} & g
\end{array}\right)\left(\begin{array}{cc}
S_{\mathrm{hh}} & S_{\mathrm{hv}} \\
S_{\mathrm{vh}} & S_{\mathrm{vv}}
\end{array}\right)\left(\begin{array}{cc}
1 & \delta_{1} \\
\delta_{2} & g
\end{array}\right)
$$

where $A$ represents the overall absolute amplitude factor, $\Phi$ represents an overall absolute phase, $\delta_{1}$ represents the crosstalk when vertically polarized ( $\delta_{2}$ when horizontally polarized) electric fields are transmitted or received, and $g$ represents the one-way copolarized channel gain imbalance in amplitude. The absolute phase $\Phi$ is lost during SAR processing and is not taken into account here. In the case of an ideal system or a perfect calibration, $A=1, \delta_{1}=\delta_{2}=0$, and $g=1$.

The elements in the matrices are complex numbers. The $\sigma^{0}$ backscattering coefficients are derived from the measured elements of the scattering matrix and are proportional to the ensemble average of the square of the modulus of these elements

$$
\sigma_{i j}^{0}=K\left\langle\left|S_{i j}\right|^{2}\right\rangle
$$

where $K$ is an overall radiometric calibration constant, which is related to $A$.
The impacts of crosstalk, channel gain imbalance, and radiometric calibration on the performance of the classification methods will be investigated separately.

\section{A. Radiometric Accuracy and Radiometric Stability}

Internal calibration is performed in real time in the system to assess $A$ and, therefore, the overall calibration constant $K$, and external calibration campaigns are carried out regularly to provide finer estimates using reference targets.

Radiometric accuracy refers to the accuracy with which the $A$ constant can be determined after a calibration campaign. It accounts for a systematic offset in measured backscatter compared to real backscatter. Typical values are below $1 \mathrm{~dB}$. It can however be deduced from (17) that the radiometric accuracy has no impact on intensity ratios, as any systematic backscatter offset will be cancelled out by the ratio.

Radiometric stability is an indicator of the backscatter variability between repeat passes due to intrinsic variations of $A$ in the system. A radiometric stability equal to $s$ implies that $A$ can vary between $A / s$ and $A \cdot s$ between two consecutive data acquisitions. The backscattering coefficients can therefore be multiplied by a factor between $1 /|s|^{2}$ and $|s|^{2}$. Typical values of $|s|^{2}$ range between 0.5 and $1 \mathrm{~dB}$.

The PR method is not affected by radiometric stability as it involves only ratios of same-date channels. For the TC method using $r_{\mathrm{TC}}=I_{p, d 2} / I_{p, d 1}$, a backscatter offset due to radiometric stability between $d 1$ and $d 2$, which is equal to $|s|^{2}$, implies that the measured ratios are equal to $|s|^{2}$ multiplied by the true ratios.

If $r_{0}$ is assessed with a supervised method, the radiometric stability has no impact on the classification error when only one couple of images is used, as the value of the retrieved threshold is affected by the same bias as the data and as the distance between classes is preserved. The multitemporal case (more than two dates), using one of the classification features presented in Section II-D1, can be impacted by radiometric stability as $\Delta r$ can be modified. The change cannot be modeled in the general case, but its impact on the error is lower than that of a change from $\Delta r$ to $\Delta r /|s|^{2}$.

On the contrary, if $r_{0}$ is assessed from other sources than the data (i.e., based on prior knowledge), the performance of the 


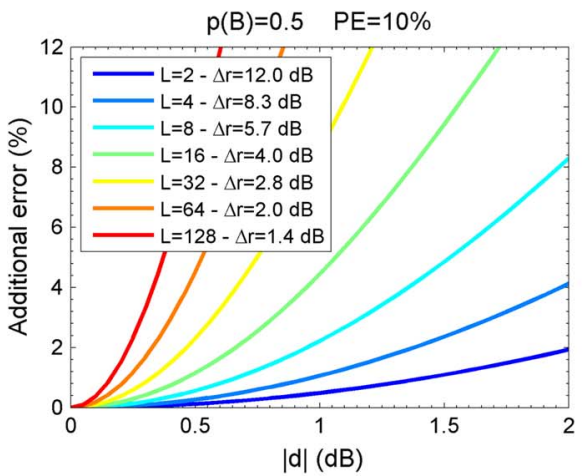

(a)

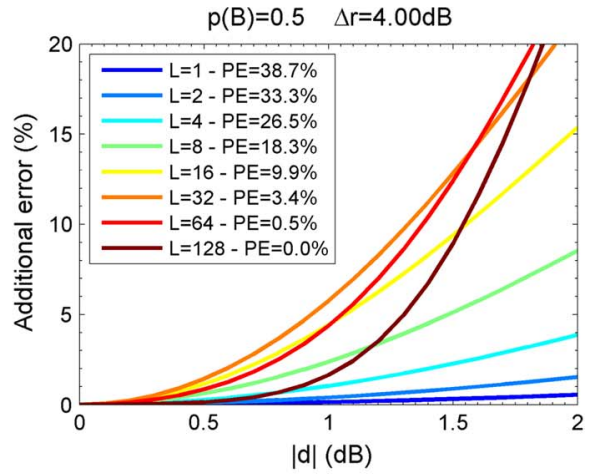

(b)

Fig. 5. (a) Additional error due to $d$ for several values of $\Delta r$ and $L$ when $P E=10 \%, d=0 \mathrm{~dB}$, and $p(B)=0.5$. (b) Probability of error as a function of $d$ for several values of $L$, with $\Delta r=10$ and $p(B)=0.5$.

classification is the same as it would be with no offset (perfect radiometric stability), and threshold $r_{t}$ is equal to $r_{0} /|s|^{2}$. Therefore, $P E$ can be assessed from (9) with $d=r_{t} / r_{0}=$ $1 /|s|^{2}$, i.e., $d_{\mathrm{dB}}=-2|s|_{\mathrm{dB}}$. In the case of range-dependent radiometric errors, i.e., when the value of $s$ varies along the range, $P E$ can be calculated locally with the corresponding value of $d$.

The present analysis is about the TC method with a single pair of dates and with an estimation of $r_{0}$ based on prior knowledge. When $p(B)=0.5$, the probability of error with $d=T$ is the same as with $d=1 / T$, so the effect of a radiometric instability that is equal to $s$ can be estimated by considering the values of $\left|d_{\mathrm{dB}}\right|$. Fig. 5 shows the additional error due to $d$ for several values of $\Delta r$ and with $L$ chosen so that they provide an error $P E$ that is equal to $10 \%$ when $d=0 \mathrm{~dB}$ and $p(B)=0.5$ [Fig. 5(a)], and it shows the additional error due to $d$ for several values of $L$, with $\Delta r=4 \mathrm{~dB}$ and $p(B)=0.5$ [Fig. 5(b)].

Among the configurations leading to $P E=10 \%$, two groups can be considered. For a small number of looks $(L \leq$ 16), corresponding to high class separability $(\Delta r \geq 4 \mathrm{~dB})$, it can be read from Fig. 5(a) that the additional error is lower than $1.2 \%$ when $\left|d_{\mathrm{dB}}\right|<0.5 \mathrm{~dB}$ (5\% when $\left|d_{\mathrm{dB}}\right|<1 \mathrm{~dB}$ ). Reversely, for high number of looks (i.e., low class separability), the sensitivity of the additional error to $d$ is very important, and $P E$ can become unacceptably high. Therefore, it is recommended to consider such classification methods based on an intensity ratio only when the class separability is high (for instance $\Delta r \geq 4 \mathrm{~dB}$ ), even though the theoretical error may be acceptable at higher number of looks for the other cases. When $\Delta r=4 \mathrm{~dB}$, Fig 5(b) shows that the additional error remains below $2 \%$ when $\left|d_{\mathrm{dB}}\right|<0.5 \mathrm{~dB}\left(6 \%\right.$ when $\left.\left|d_{\mathrm{dB}}\right|<1 \mathrm{~dB}\right)$ and that the additional error decreases when $L$ increases for very high values $(L>32)$.

In both cases (low and high number of looks), if $\Delta r \geq 4 \mathrm{~dB}$, the radiometric stability should not be a decisive parameter when its value is not too high. However, it could contribute to a nonnegligible additional error (around 6\%) under some unfavorable conditions $\left(\left|d_{\mathrm{dB}}\right|=1 \mathrm{~dB}, L \approx 30\right)$. Nevertheless, when one tries to discriminate two close classes $(\Delta r<4 \mathrm{~dB})$ through the use of a high number of looks, a degradation of the performance is noticeable. This can affect, for example, the accurate classification with high-resolution SARs, for which the requirement on radiometric stability may have to be more stringent.

\section{B. Channel Gain Imbalance}

Channel gain imbalance is a measure of the accuracy of the intensity in one channel (polarization) relative to another. It expresses radiometric errors between polarization channels. The effect of channel gain imbalance on the scattering matrix is derived from (16) when $\delta_{1}=\delta_{2}=0$

$$
\left(\begin{array}{cc}
Y_{\mathrm{hh}} & Y_{\mathrm{hv}} \\
Y_{\mathrm{vh}} & Y_{\mathrm{vv}}
\end{array}\right)=\left(\begin{array}{cc}
S_{\mathrm{hh}} & g \cdot S_{\mathrm{hv}} \\
g \cdot S_{\mathrm{vh}} & g^{2} \cdot S_{\mathrm{vv}}
\end{array}\right)
$$

Obviously, the TC method is not affected by channel gain imbalance as it involves only one polarization.

It can be derived from (17) and (18) that a PR between the two copolarized channels ( $\mathrm{HH}$ and VV) is affected by an offset that is equal to $|g|^{4}$ or $1 /|g|^{4}$ and by an offset that is equal to $|g|^{2}$ or $1 /|g|^{2}$ for the PR of one copolarized channel and one cross-polarized channel (HV or VH). The typical value of $|g|^{4}$ is below $0.5 \mathrm{~dB}$ for most systems.

When using the single-date classification feature $r_{\mathrm{PR}}=$ $I_{p 2, d} / I_{p 1, d}$ with an estimation of $r_{0}$ based on prior knowledge, $P E$ can be assessed from (9), with $d= \pm 4|g|_{\mathrm{dB}}$ for the ratio of the copolarizations or $d= \pm 2|g|_{\mathrm{dB}}$ if one cross-polarization is involved. In this case, it can be deduced from Fig. 5 that the channel gain imbalance should not be a decisive parameter, provided that its value remains within the usual range $\left(\left|d_{\mathrm{dB}}\right|<\right.$ $0.5 \mathrm{~dB}$ or $\left.\left|d_{\mathrm{dB}}\right|<0.25 \mathrm{~dB}\right)$. However, similar to the TC, the case of close classes discriminated through the use of a high number of looks is very sensitive to channel gain imbalance, and it leads to more stringent requirements, especially when considering the ratio of two copolarizations.

If $r_{0}$ is assessed with a supervised method, the channel gain imbalance has no impact on the classification error in the single-date case as the value of the retrieved threshold is affected by the same gain imbalance since the data and the distance between classes are preserved. In the multidate case, with the feature described in Section II-D2, $\Delta r$ could be, in 
theory, slightly modified by channel gain imbalance, provided that the latter is not stable in time, depending on the temporal behavior of the PR of the two classes. However, given the low probability of occurrence of such unfavorable conditions and the low impact that they would have on the error, the overall effect is negligible.

\section{Crosstalk}

Crosstalk represents the channel isolation. It is a measure of the intensity in the polarization which is orthogonal to the one that is intended to be transmitted or received.

We simplify the model presented in (16) by considering that the crosstalk has the same value in both vertical and horizontal channels: $\delta_{1}=\delta_{2}=\delta$. Values of $|\delta|_{\mathrm{dB}}$ below $-30 \mathrm{~dB}$ (i.e., $|\delta|=0.032=10^{-30 / 20}$ ) are now readily achieved with satellite SARs. When $g=1$, the measured scattering matrix is given in (19), shown at the bottom of the page.

It is assumed that $S_{\mathrm{hv}}=S_{\mathrm{vh}}$.

In theory, one needs to know the values of the three polarizations in order to assess the impact of crosstalk on classification methods based on TC and PR. It is, therefore, difficult to precisely address this issue in the general case. We can however investigate the magnitude of the perturbation caused by crosstalk in each channel by considering the following: 1) The HH and VV backscatters are usually of the same order of magnitude on natural targets, and they would not differ by more than $8 \mathrm{~dB}$, so $\left|S_{\mathrm{HH}}\right|=a\left|S_{\mathrm{VV}}\right|$, with $1 / 2.5<a<2.5$, and 2) $\mathrm{HV}$ is often one order of magnitude lower than the copolarized channels (e.g., between 3 and $12 \mathrm{~dB}$ lower), so $\left|S_{\mathrm{HH}}\right|=b\left|S_{\mathrm{HV}}\right|$, and $\left|S_{\mathrm{VV}}\right|=c\left|S_{\mathrm{HV}}\right|$, with $1.4<b<4$ and $1.4<c<4$.

The percent perturbation in amplitude caused by crosstalk in copolarized channels is

$$
\begin{aligned}
\Delta A_{\mathrm{copol}} & =\frac{\left|2 \delta S_{\mathrm{hv}}+\delta^{2} S_{\mathrm{vv}}\right|}{\left|S_{\mathrm{hh}}\right|} \\
& \leq \frac{2|\delta|\left|S_{\mathrm{hv}}\right|+|\delta|^{2}\left|S_{\mathrm{vv}}\right|}{\left|S_{\mathrm{hh}}\right|} \\
& =2 \frac{|\delta|}{b}+\frac{|\delta|^{2}}{a} \\
& \leq 1.43|\delta|+2.5|\delta|^{2} .
\end{aligned}
$$

When $|\delta|$ is equal to $-30 \mathrm{~dB}, \Delta A_{\text {copol }}$ is lower than $4.8 \%$, which would lead to a maximum backscatter perturbation of $0.4 \mathrm{~dB}$. A copolarized intensity ratio could be offset by a maximum value of $0.8 \mathrm{~dB}$ in the worst case. The corresponding additional error is shown in Fig. 7, with $|d|=0.8 \mathrm{~dB}$, and it is lower than $4 \%$ when $\Delta r>4 \%$. The effect of crosstalk is, therefore, relatively negligible in copolarized channels.
In cross-polarized channels, the percent perturbation in amplitude is

$$
\begin{aligned}
\Delta A_{\text {cross-pol }} & =\frac{\left|\delta S_{\mathrm{hh}}+\delta S_{\mathrm{vv}}+\delta^{2} S_{\mathrm{hv}}\right|}{\left|S_{\mathrm{hv}}\right|} \\
& \leq \frac{|\delta|\left|S_{\mathrm{hh}}\right|+|\delta|\left|S_{\mathrm{vv}}\right|+|\delta|^{2}\left|S_{\mathrm{hv}}\right|}{\left|S_{\mathrm{hv}}\right|} \\
& =|\delta|(b+c)+|\delta|^{2} \\
& \leq 8|\delta|+|\delta|^{2} .
\end{aligned}
$$

When $|\delta|$ is equal to $-30 \mathrm{~dB}, \Delta A_{\text {cross-pol }}$ can reach $26 \%$, which could lead to a backscatter perturbation as high as $2 \mathrm{~dB}$. The intensity ratio of one copolarized channel and one crosspolarized channel can therefore be around $2.4 \mathrm{~dB}$ in unfavorable cases and up to $4 \mathrm{~dB}$ for a temporal ratio of cross-polarized intensities. The use of cross-polarizations in intensity ratios should therefore be subject to very severe requirements on crosstalk. For example, a crosstalk value lower than $-40 \mathrm{~dB}$ would guarantee that the backscatter perturbation is lower than $0.6 \mathrm{~dB}$ for cross-polarizations.

In conclusion, in classification methods based on an intensity ratio, crosstalk does not seem to be an issue as long as only copolarizations are dealt with. However, as expected, it may be very critical when cross-polarizations are involved.

\section{IMPACt OF Other System PARAMETERS}

Apart from calibration parameters, other mission and system parameters (satellite repeat cycle, spatial resolution, and ambiguity ratio) can affect the classification accuracy, but their effect cannot be assessed in the general case. They have to be considered together with application- and scene-specific parameters, e.g., the temporal backscattering profile of the two classes when dealing with multitemporal ratios, the presence of targets with high backscatter that would maximize error due to ambiguity, or the typical size of patches.

\section{A. Impact of Ambiguity}

Ambiguity is a form of ghosting that happens when bright targets are illuminated by the sidelobes of the SAR antenna and contaminate the backscattering return attributed to neighboring areas illuminated by the main lobe. Range ambiguity occurs from ambiguous zones whose slant range differs from that of the desired zone by nonzero multiples of the pulse repetition distance and whose Doppler frequencies differ by multiples of the PRF [25]. Azimuth ambiguity is caused by zones whose slant ranges are the same as the desired zone, but whose Doppler frequencies differ by multiples of the PRF [25]. The distributed target ambiguity ratio is the ratio of the unwanted ambiguous intensity to the wanted target intensity, taking into

$$
\left(\begin{array}{cc}
Y_{\mathrm{hh}} & Y_{\mathrm{hv}} \\
Y_{\mathrm{vh}} & Y_{\mathrm{vv}}
\end{array}\right)=\left(\begin{array}{cc}
S_{\mathrm{hh}}+2 \delta S_{\mathrm{hv}}+\delta^{2} S_{\mathrm{vv}} & \left(1+\delta^{2}\right) S_{\mathrm{hv}}+\delta\left(S_{\mathrm{hh}}+S_{\mathrm{vv}}\right) \\
\left(1+\delta^{2}\right) S_{\mathrm{hv}}+\delta\left(S_{\mathrm{hh}}+S_{\mathrm{vv}}\right) & S_{\mathrm{vv}}+2 \delta S_{\mathrm{hv}}+\delta^{2} S_{\mathrm{hh}}
\end{array}\right)
$$


account both range and azimuth ambiguity. Typical values range from -17 to $-40 \mathrm{~dB}$.

The impact of ambiguity may be expressed in the following terms. With the ambiguity ratio noted as $a$, the measured complex amplitude $S_{a}$ relates to the complex amplitude of the observed scene $S_{0}$ and to the complex amplitude of the scene at the source of the ambiguity $S_{s}$ by the following relationship:

$$
S_{a}=S_{0}+\sqrt{a} S_{s} .
$$

The relationship that is equivalent to (20) for the backscatter intensity is retrieved using (17)

$$
I_{a}=K\left\langle\left|S_{0}^{2}+a S_{s}^{2}+2 \sqrt{a} S_{0} S_{s}\right|\right\rangle \leq I_{0}+a I_{s}+2 \sqrt{a I_{0} I_{s}} .
$$

The ambiguities increase the measured backscatter, and the effect is more important when the backscatter of the ambiguous area is high compared to the backscatter of the observed area. Therefore, the change in backscatter is the most critical when the source of the ambiguity is the brightest elements in the scene, e.g., built-up areas.

To quantify the impact of ambiguity on the classification performance, one has to assess the changes in the class parameters $r_{A}$ and $r_{B}$ that are caused by the ambiguity. For that purpose, the original backscatter values $I_{1}$ and $I_{2}$ composing the intensity ratio $r=I_{2} / I_{1}$ need to be known for the two classes. For simplicity, we suppose here that $r_{A}=0 \mathrm{~dB}$, so $I_{1, A}=I_{2, A}$. This is quite realistic for the TC method (class A being usually assumed stable in time) and for the PR method in the case of a ratio of copolarized intensities (copolarizations being of the same order of magnitude in many natural targets).

Under this assumption, the backscatter increase that is due to ambiguity is the same in $I_{1, A}$ and $I_{2, A}$, and $r_{A}$ remains to be equal to $0 \mathrm{~dB}$. The backscatter values of class B are $I_{1, B}$ and $I_{2, B}=\left(I_{1, B}+\Delta r\right) \mathrm{dB}$.

The effect of ambiguity on $I_{1, B}$ and $I_{2, B}$ is calculated from (21). We simulate here the worst case when the ambiguous source is an urban area and when the ambiguous backscatter equals the right-hand side of the inequality in (21). The default backscattering value taken for urban areas is $I_{s}=0 \mathrm{~dB}$ at any date and polarization. The modified values of $I_{1, B}$ and $I_{2, B}$ lead to a modified intensity ratio $r_{B}$ and to a new value of the $\Delta r$ parameter, noted as $\Delta r_{a}$.

Fig. 6 shows the value of the class separability, with ambiguity $\left(\Delta r_{a}\right)$ as a function of the ambiguity ratio $(a)$, for several values of $I_{1, B}$ and for an initial class separability $\Delta r=8 \mathrm{~dB}$. As expected, the effect of ambiguities is higher when class $\mathrm{B}$ has low backscatter values. When $I_{1, B}=-16 \mathrm{~dB}, \Delta r_{a}$ can decrease below $5 \mathrm{~dB}$ in extreme cases $(a=-17 \mathrm{~dB})$.

The reduction in $\Delta r_{a}$ leads to an increase of the classification error. Fig. 7 shows the effect of the ambiguity ratio on the additional classification error due to ambiguity for the case when $I_{1, B}=-10 \mathrm{~dB}, \Delta r=8 \mathrm{~dB}$, and $p(B)=0.5$ and for several numbers of looks $L$. For the worst case of an ambiguity ratio that is equal to $-17 \mathrm{~dB}$, the additional error is higher than $4 \%$ for a low number of looks $(L<16)$ and can rise up to $6 \%$, but it is negligible when $L$ is above 30 .

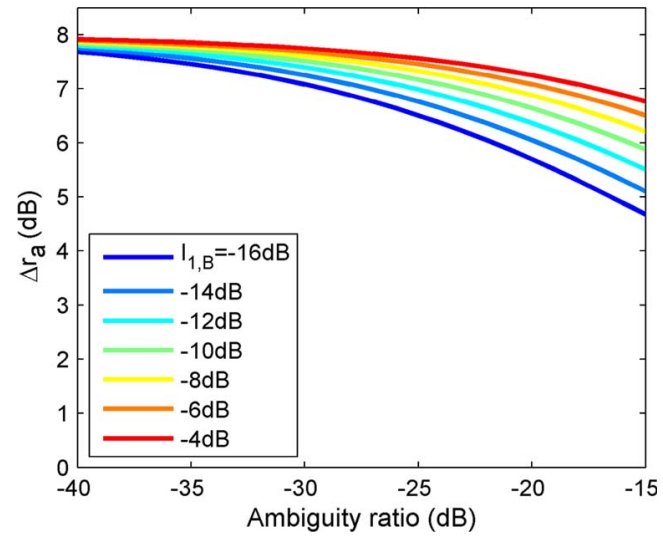

Fig. 6. Effect of the ambiguity ratio $a$ on $\Delta r_{a}$ for several values of $I_{1, B}$.



Fig. 7. Effect of $a$ on the additional error due to ambiguity.
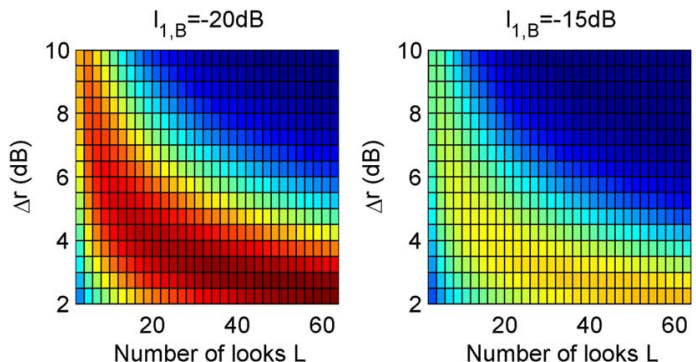

$I_{1, B}=-10 \mathrm{~dB}$


Fig. 8. Additional classification error due to ambiguity as a function of $\Delta r$ and $L$ for four values of $I_{1, B}$.

The error also varies with $\Delta r$ and $I_{1, B}$. Fig. 8 shows the additional error when $a=-17 \mathrm{~dB}$ for four values of $I_{1, B}$ from -20 to $-5 \mathrm{~dB}$, as a function of $\Delta r$ and $L$. For sufficiently high values of both $\Delta r$ and $L$, the additional error is negligible (below $2 \%)$. When the class separability is poor $(\Delta r<4 \mathrm{~dB})$, the additional error is high and, somehow paradoxically, increases 
with the number of looks. When the number of looks is low (below 15), the classification performance is highly sensitive to ambiguity, even for high class separability.

In summary, ambiguities can have a critical impact on the performance of the classification methods when the ambiguity ratio is high $(a=-17 \mathrm{~dB})$ if the class separability is low and/or if the number of looks is low. For a given SAR system, the methods should therefore be applied only when the classes are highly separable. Further simulations show that, when the ambiguity is better than $-30 \mathrm{~dB}$, the additional error is however limited to $6 \%$ in the very worst case, including poorly separable classes.

\section{B. Impact of the Temporal Sampling}

In both methods, it is generally implicitly supposed that the class separability, measured by $\Delta r$, is due mostly to the outstanding behavior of the intensity ratio (TC or PR) of one class of interest, which reaches peculiar values in time, while the intensity ratio of the other class remains relatively constant around its typical value. We suppose here that the class of interest is class $\mathrm{B}$ and that its intensity ratio is remarkable because of its high values $\left(r_{B}>r_{A}\right)$.

In some applications, the outstanding radiometric behavior of class B is caused by a point event, and it lasts forever after the event. In that case, the timing of the data acquisitions is not very important, provided that one date is available after the event for the PR method and one date before and one after for the TC method. Events such as deforestation or urbanization would be illustrative of this category.

In other situations, the outstanding behavior of class B is caused by a phenomenon, which lasts for a finite period during which the intensity ratio of class B expresses its particularity and then stops. Applications such as crop or flood monitoring are concerned on this approach. The $\Delta r$ parameter then represents the theoretical optimal class separability obtained when the data are acquired at the optimal dates. In the multitemporal case introduced in Section II-D, the value of the observed class separability will depend on the timing of the available acquisitions and on the temporal backscattering profiles of the two classes during the period when the phenomenon occurs. The temporal sampling of the acquisitions is an important parameter in this case, as a high observation frequency will increase the probability to have optimal dates in the available data set.

In this section, temporal backscattering profiles are modeled to simulate the typical behavior of the two classes that are likely to be involved in classification schemes based on the TC or the PR. These modeled profiles are then used to assess the effect of the observation frequency on the classification performance.

1) Theoretical Data Model: We model here the backscattering profiles of the two classes during the period when the phenomenon occurs, which is assumed to last for $c$ days.

a) TC method: We illustrate the case corresponding to class B to a temporal backscattering increase, i.e., a positive intensity ratio. Therefore, the multitemporal classification feature is $r_{\mathrm{TC}, \text { multi }}=\max _{i, j>i}\left[I_{p, d j} / I_{p, d i}\right]$, where $p$ is the polarization and $(d i)_{i=1: N}$ represents the dates in the available time series, as suggested in Section II-D1.
As suggested in Section IV-A, the backscatter intensity for class A is supposed to be constant during the $c$ days of the considered phenomenon. Therefore, $r_{A, \mathrm{TC}}=0 \mathrm{~dB}$, and $\Delta r_{\mathrm{TC}}=$ $r_{B, \mathrm{TC}}$. The backscatter of class $\mathrm{B}$ is modeled by a function that increases from $-10 \mathrm{~dB}$ to $-10+\Delta r_{\mathrm{TC}} \mathrm{dB}$ during $c$ days. For a day $D$ during this period, between day 0 and day $c$, the backscatter intensity of class $\mathrm{B}$ at the polarization $p$ is given by

$$
\begin{aligned}
I_{p, D}=-10+\Delta r_{\mathrm{TC}} \cdot(1+\exp ( & \left.-\frac{10 D}{c}\right) \\
& \left.-2 \exp \left(-\frac{5 D}{c}\right)\right)
\end{aligned}
$$

(all the values are expressed in decibels).

b) PR method: The multitemporal classification feature for the PR method is $r_{\mathrm{PR}, \operatorname{multi}}=\max _{i}\left[I_{p 2, d i} / I_{p 1, d i}\right]$, where $p 1$ and $p 2$ are the two polarizations and $(d i)_{i=1: N}$ represents the dates in the available time series. We suppose that the PR of class A remains stable in time at a constant value $r_{A, P R}$. Again, this value is taken to be equal to $0 \mathrm{~dB}$, leading to $\Delta r_{\mathrm{PR}}=r_{B, \mathrm{PR}}$. The $\mathrm{PR}$ of class $\mathrm{B}$ is modeled by a function that increases from 0 to $\Delta r_{\mathrm{PR}} \mathrm{dB}$ during the first half of the period during which the phenomenon lasts (day $0-c / 2$ ) and then decreases back to $0 \mathrm{~dB}$ during the second half of the period (day $c / 2-c)$.

The PR of class $\mathrm{B}$ is given by

$$
\begin{aligned}
\frac{I_{p 2, D}}{I_{p 1, D}} & =\Delta r_{\mathrm{PR}}\left[1+\exp \left(-\frac{20 D}{c}\right)-\exp \left(-\frac{20(c-D)}{c}\right)\right. \\
& \left.-2\left(\exp \left(-\frac{10 D}{c}\right)-\exp \left(-\frac{10(c-D)}{c}\right)\right)\right]
\end{aligned}
$$

When $r_{A, \mathrm{PR}}$ is not equal to $0 \mathrm{~dB}, \Delta r_{\mathrm{PR}}$ should be replaced by $\Delta r_{\mathrm{PR}}+r_{A, \mathrm{PR}}$ in (23).

Fig. 9 shows the temporal behavior of the backscattering coefficient and of the PR of class B corresponding to the TC and PR methods, respectively, when $\Delta r=8 \mathrm{~dB}$ and when the phenomenon causing the distinctive behavior of class $B$ lasts for 80-120 days.

For the TC method, the backscatter of class B is shown in Fig. 9 with a value of $-10 \mathrm{~dB}$ for the dates outside of the event $(D<0$ and $D>c$ ). The drastic backscatter change (here, a decrease) at the end of the event is a representative, for example, of harvest in crop monitoring applications. However, it has no consequence in this paper as it is supposed that the SAR data are acquired only during the phenomenon $(0 \leq D \leq c)$.

2) Model Results: The parameters $\Delta r_{\mathrm{TC}}$ and $\Delta r_{\mathrm{PR}}$ used in (22) and (23) represent the optimal class separability in the TC and PR methods, which is obtained when the data are acquired at the optimal dates, i.e., one date on the first day and one on the last day of the period for the TC method, and one date at the mid-period for the PR method. In practice, the data can be acquired only at a limited number of dates when the area of interest is visible by the SAR instrument, which depends on the satellite orbit. The observed class separability is, therefore, lower than its theoretical value.

It is considered here that the observation frequency is equal to the time-lapse between satellite repeat-pass orbits, which corresponds to the maximum acquisition frequency that is achievable 



Fig. 9. Temporal evolution of (left) the backscattering coefficient and (right) the PR of class B when $\Delta r=8 \mathrm{~dB}$.
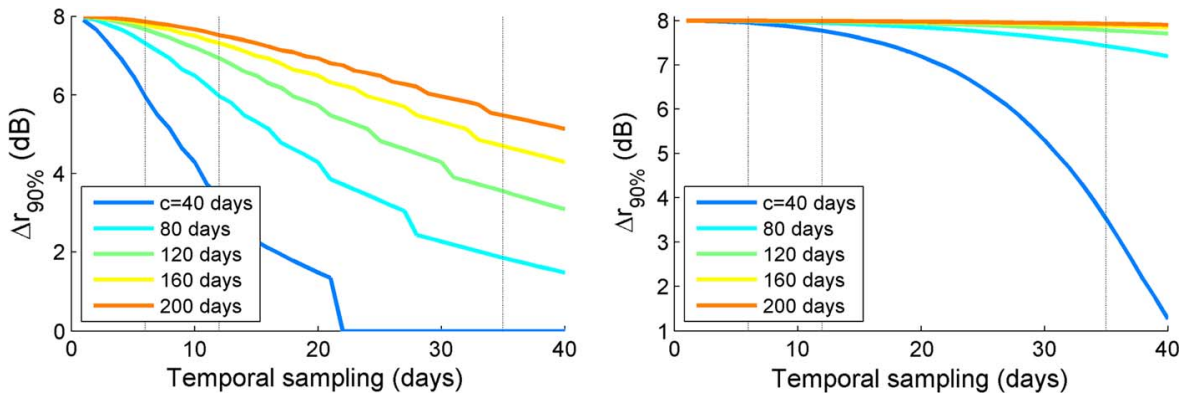

Fig. 10. $\Delta r_{90 \%}$ parameter as a function of $c$ for different values of $f$ for the (left) TC and (right) PR methods, with $\Delta r=8 \mathrm{~dB}$.

under a fixed incidence angle. More frequent observations of a single area can be made by using multi-incidence data sets or different subsets of overlapping images from adjacent satellite tracks, but in both cases, the local incidence angle will change from one image to the other, which can be a severe limitation as the backscattering profiles vary with the incidence. Moreover, when classifying a large area at a regional to continental scale, multi-incidence data sets cannot be made available because of acquisition conflicts between subsets of the area that could be illuminated simultaneously by the instrument at different incidence angles.

The temporal sampling of the acquisitions has a direct impact on the observed class separability. Indeed, the more often the acquisitions take place, the more likely it is to catch a high value of the intensity ratio for class B and, therefore, to maximize its classification feature $r_{B}$ and, consequently, the class separability $\Delta r$.

Based on the temporal profiles of the backscattering and of the PR given in (22) and (23), we can compute the value of the $\Delta r$ parameter for a data set of several images acquired every $f$ days during the $c$ days corresponding to the full duration of the phenomenon. The value of $\Delta r$ depends on the time of the first acquisition, which is between days 1 and $f$ after the beginning of the phenomenon. Therefore, the value of $\Delta r$ is calculated for all $f$ cases that can be encountered accordingly to the date of the first acquisition. To provide an estimation of $\Delta r$, we derive the $\Delta r_{90 \%}$ parameter corresponding to the value above which $\Delta r$ is found in $90 \%$ of the possible cases. $\Delta r_{90 \%}$ is used as a proxy for $\Delta r$.

Fig. 10 shows the values of $\Delta r_{90 \%}$ for different values of $c$ and $f$ for the TC (left) and PR (right) methods when $\Delta r=8 \mathrm{~dB}$.

Particular values of $f$ are highlighted in Fig. 10, corresponding to ASAR onboard ENVISAT ( $f=35$ days), Sentinel-1
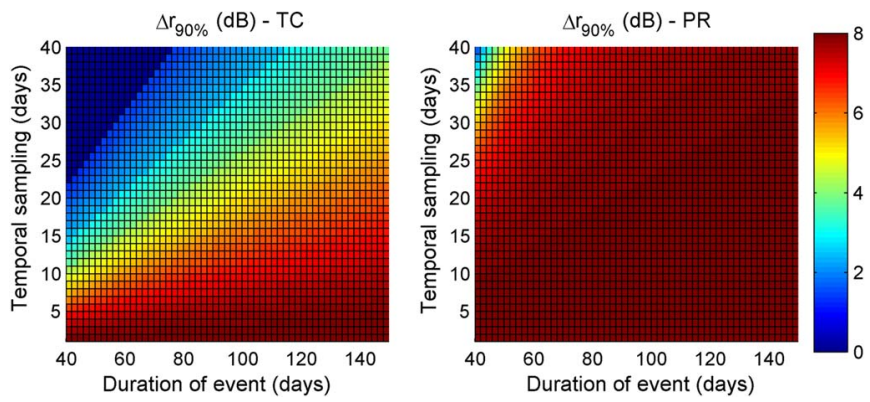

Fig. 11. $\Delta r_{90 \%}$ parameter as a function of $c$ and $f$ for the (left) TC and (right) PR methods.

with one satellite only ( $f=12$ days), and Sentinel-1 in a constellation of two satellites ( $f=6$ days).

Fig. 11 shows the values of $\Delta r_{90 \%}$ for a wide range of values of $c$ and $f$ for both methods when $\Delta r=8 \mathrm{~dB}$.

The impact of the temporal sampling is small in the PR method. $\Delta r_{90 \%}$ remains above $7 \mathrm{~dB}$ for most configurations, except when the duration of the phenomenon is close to the temporal sampling ( $c<60$ days and $f>30$ days). The classification accuracy corresponding to $\Delta r=7 \mathrm{~dB}$ and $\Delta r=8 \mathrm{~dB}$ is $96.0 \%$ and $97.7 \%$, respectively, when the number of looks is $L=10$.

On the contrary, $f$ is a critical parameter for the TC method, especially for short-duration phenomena. When the temporal sampling is not high enough compared to the duration of the phenomenon, the classes are not separable at all $\left(\Delta r_{90 \%}=\right.$ $0 \mathrm{~dB})$. For a phenomenon lasting for 100 days, $\Delta r_{90 \%}$ increases from 2.4 to 6.6 and $7.5 \mathrm{~dB}$ when the temporal sampling increases from every 35 to every 12 and 6 days, which corresponds to a classification accuracy of $72.9 \%, 95.1 \%$, and $97.0 \%$, respectively, when $L=10$. 


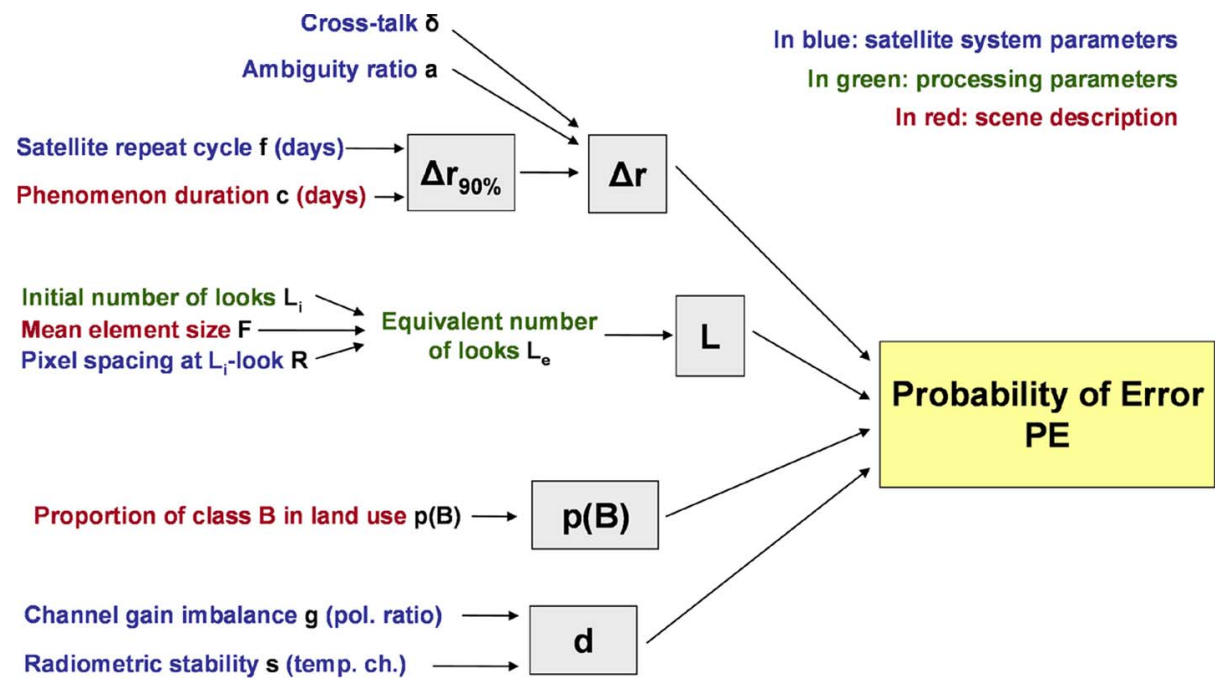

Fig. 12. Effects of the (in blue) satellite system parameters, (in green) image processing parameters, and (in red) scene parameters on the overall probability of error in mapping performance. The intermediary parameters are in gray boxes.

\section{Relationship Between the Mean Size of Significant Elements, the Spatial Resolution, and the Equivalent Number of Looks}

Space agencies usually deliver multilooked intensity products to users, with an initial number of looks that is equal to $L_{i}$. If $L_{i}$ is too low for the considered applications, users can perform a spatial multilooking to raise the number of looks from $L_{i}$ to $L_{e}$, which is the equivalent number of looks. This consists in associating a unique pixel to any batch of $N \times N L_{i}$-look pixels, where the intensity of this pixel is the mean intensity of the pixels in the batch. Of course, the image definition is degraded by a factor $N$. In SAR images, the number of independent samples in a population of $X$ samples is found between $X / 4$ and $X / 2$ [26]. Therefore, the resulting equivalent number of looks $L_{e}$ is such that

$$
N^{2} L_{i} / 4<L_{e}<N^{2} L_{i} / 2 \text {. }
$$

The spatial multilooking level should be related to the mean dimension $F$ of the significant elements in the observed scene (for example, fields, forest stands, and ice floes) and to the initial pixel spacing $R$ at $L_{i}$-look so that the multilooking step does not involve summing over too heterogeneous areas. For example, it can be decided that $R \times N<(F / 2)$ (the same inequality applies for most speckle filters based on $N \times N$ neighboring windows), which leads to limitations in the final number of looks

$$
L_{e}<F^{2} L_{i} / 8 R^{2}
$$

or to requirements on the spatial resolution

$$
R<F / \sqrt{8 L_{e} / L_{i}} .
$$

Hence, for a given $L_{i}$-look spatial resolution $R$ and a given typical size $F$ of the observed elements, one can derive the maximum equivalent number of looks to be used in Fig. 3 by applying (25).

\section{Summary of the Results}

The following key parameters for this analysis have been identified.

1) Satellite system parameters:

a) satellite repeat cycle $\mathbf{f}$ (in days);

b) pixel spacing of an $L_{i}$-look product $\mathbf{R}$ (in meters);

c) distributed target ambiguity ratio a (in decibels);

d) channel gain imbalance g (in decibels)—for the PR method only;

e) radiometric stability $\mathbf{s}$ (in decibels)—for the $\mathrm{TC}$ method only.

2) Processing parameters (multilooking):

a) initial number of looks of the product $\mathbf{L}_{\mathbf{i}}$;

b) equivalent number of looks after spatial multilooking $\mathbf{L}_{\mathbf{e}}$.

3) Scene description parameters:

a) mean size of the observed elements $\mathbf{F}$ (in meters);

b) duration of the monitored phenomenon $\mathbf{c}$ (in days);

c) proportion of class $B$ in land use $\mathbf{p}(\mathbf{B})$ (in percent).

Fig. 12 shows the relations between these parameters and the intermediary parameters introduced in Section II (namely, $L, \Delta r, p(B)$, and $d$ ), as it has been discussed in the two last sections.

Table I summarizes the effects of these parameters on the probability of error of the classifications.

\section{EXPERIMENTAL VALIDATION: COMPARISON WITH REAL DATA}

In order to assess the validity of the model presented in this paper, some of its outputs should be confronted with experimental results from real SAR data. Rice field classification is a convenient application for this purpose as it can illustrate both the TC and PR methods. It was shown in the past studies that rice fields are characterized by a high temporal 
TABLE I

Impacts of (Top) Satellite System Parameters, (Middle) Image Processing Parameters, and (Bottom) Scene Parameters on the Overall Performance of the Mapping Algorithms Based on Intensity Ratio Methods (Temporal Change and Polarization Ratio Methods)

\begin{tabular}{|c|c|c|}
\hline Input parameters & $\begin{array}{l}\text { Intermediary } \\
\text { parameter }\end{array}$ & Impact \\
\hline Satellite repeat cycle $\mathbf{f}$ (days) & $\Delta \mathrm{r}$ & $\begin{array}{l}\text { TC: a low } \mathrm{f}(6 \text { or } 12 \text { days) increases significantly the mapping } \\
\text { accuracy compared to higher values ( } 35 \text { days). PR: f is not } \\
\text { critical. }\end{array}$ \\
\hline $\begin{array}{l}\text { Pixel spacing } \mathbf{R} \text { of a L-look } \\
\text { product }(\mathrm{m})\end{array}$ & $\mathrm{L}$ & $\begin{array}{l}\text { A lower } \mathrm{R} \text { will allow a higher equivalent number of looks } \\
\text { after multi-looking, thus a smaller error. }\end{array}$ \\
\hline $\begin{array}{l}\text { Distributed ambiguity ratio } \\
\qquad(\mathrm{dB})\end{array}$ & $\Delta \mathrm{r}$ & $\begin{array}{l}\text { Additional error is small }(<6 \%) \text { if } a<30 \mathrm{~dB} \text {, but can be critical } \\
\text { for } \mathrm{a}=-17 \mathrm{~dB} \text { and a low } \Delta \mathrm{r} \text { and/or } \mathrm{L} \text {. }\end{array}$ \\
\hline $\begin{array}{l}\text { Channel gain imbalance } \mathbf{g} \\
\quad(\mathrm{dB})\end{array}$ & $\mathrm{d}$ & $\begin{array}{l}\text { PR: In the nominal case }(|\mathrm{d}| \leq 0.5 \mathrm{~dB}) \text {, the additional error is } \\
\text { negligible }(<1 \%) \text {. TC: No impact. }\end{array}$ \\
\hline Radiometric stability $\mathbf{s}(\mathrm{dB})$ & d & $\begin{array}{l}\text { TC: In the least favourable range of the nominal case } \\
(|\mathrm{d}| \approx 1 \mathrm{~dB}) \text {, the additional error can be non-negligible }(\approx 6 \%) \text {. } \\
\text { PR: No impact. }\end{array}$ \\
\hline Cross-talk $\boldsymbol{\delta}(\mathrm{dB})$ & $\Delta \mathrm{r}$ & $\begin{array}{l}\text { Very strong impact on cross-polarizations (needs to be lower } \\
\text { than }-40 \mathrm{~dB} \text { to limit the additional error to } 6 \% \text { in all cases). }\end{array}$ \\
\hline Initial number of looks $\mathbf{L}_{\mathbf{i}}$ & $\mathrm{L}$ & $\begin{array}{l}\text { A higher } L_{i} \text { will provide a higher } L \text { and thus reduce the } \\
\text { probability of error. }\end{array}$ \\
\hline $\begin{array}{l}\text { Equivalent number of looks } \\
\mathbf{L}_{\mathbf{e}} \text { after spatial multilooking }\end{array}$ & $\mathrm{L}$ & $\begin{array}{l}\text { A higher } L_{e} \text { will provide a higher } L \text { and thus reduce the } \\
\text { probability of error. However } L_{e} \text { is constrained by } F \text { and } R \text {. }\end{array}$ \\
\hline Mean element size $\mathbf{F}(\mathrm{m})$ & $\mathrm{L}$ & $\begin{array}{l}\text { A higher } \mathrm{F} \text { will allow a higher equivalent number of looks } \\
\text { after multi-looking, thus a smaller error. }\end{array}$ \\
\hline $\begin{array}{l}\text { Duration of phenomenon } \mathbf{c} \\
\text { (days) }\end{array}$ & $\Delta \mathrm{r}$ & $\begin{array}{l}\text { TC: the error increases with decreasing phenomenon duration, } \\
\text { reinforcing the need for a small f. PR: c is not critical. }\end{array}$ \\
\hline $\begin{array}{l}\text { Proportion of class B in land } \\
\text { use } \mathbf{p}(\mathbf{B})(\%)\end{array}$ & $\mathrm{p}(\mathrm{B})$ & $\begin{array}{l}\text { If } \mathrm{d}=0 \mathrm{~dB} \text { (no channel gain imbalance, no radiometrio } \\
\text { stability), } \mathrm{p}(\mathrm{B}) \text { has no impact on the error. Otherwise, the } \\
\text { additional error compared to the default value is negligible } \\
(<2 \%) \text { for }|\mathrm{d}| \leq 0.5 \mathrm{~dB} \text {. }\end{array}$ \\
\hline
\end{tabular}

backscatter increase during the rice season at $\mathrm{HH}$ and $\mathrm{VV}$ polarizations and by a PR HH/VV that reaches high values during the season compared to other land use classes (see, for example, the references listed in [16]). We suggest here to use the HH backscatter TC for the TC method and the HH/VV backscatter ratio for the PR method. In the past experiments, it was found that, in rice growing regions, the other land cover types are usually tree plantations, perennial crops, or urban/man-made areas having low backscatter TC. For most of those nonrice classes, it is also true that $\mathrm{HH} \approx \mathrm{VV}$. Therefore, for both TC and PR methods, class A is nonrice, and class B is rice $\left(r_{A}<r_{B}\right)$. However, some land use classes should sometimes be masked out, e.g., using geographic information systems (GIS) data, like water surfaces with unpredictable changes in the TC method or urban/man-made areas, which can also be characterized by a strong HH/VV PR, in the PR method.

We have acquired three images from the ASAR instrument onboard ENVISAT, under the alternating polarization acquisition mode with polarizations $\mathrm{HH}$ and $\mathrm{VV}$, over a rice-growing region in Vietnam. The three dates cover a whole rice growth period and are separated by $f=35$ days, which corresponds to the satellite repeat-pass period. Short-cycle rice varieties are grown in this area, with a growing period lasting for about $c=80$ days.

The data are ordered in single-look complex (SLC) format, providing the complex amplitudes at each pixel in slant-range geometry, and in precision image (PRI) format, providing multilook intensities in ground-range geometry.

An ancillary GIS data set depicting rice and nonrice areas is available over a small region and is used to plot the histograms of the intensity ratio of each class for each method and to retrieve the corresponding class parameters, as described in [16]. The multitemporal approach is opted for. For the PR method with $r_{\mathrm{PR}}=\max _{i=1,2,3}\left[\left(\sigma_{\mathrm{HH}}^{0} / \sigma_{\mathrm{VV}}^{0}\right)_{d i}\right]$, the class parameters are found to be $r_{A, \mathrm{PR}}=0.87 \mathrm{~dB}$ and $r_{B, \mathrm{PR}}=7.44 \mathrm{~dB}$; therefore, $\Delta r_{\mathrm{PR}}=6.57 \mathrm{~dB}$. For the TC method with $r_{\mathrm{TC}}=$ $\max _{i=1,2,3, j>i}\left[\left(\sigma_{\mathrm{HH}}^{0}\right)_{d j} /\left(\sigma_{\mathrm{HH}}^{0}\right)_{d i}\right]$, the class parameters are $r_{A, \mathrm{TC}}=1.43 \mathrm{~dB}$ and $r_{B, \mathrm{TC}}=2.82 \mathrm{~dB}$; therefore, $\Delta r_{\mathrm{TC}}=$ $1.39 \mathrm{~dB}$. Considering that these are the $\Delta r_{90 \%}$ parameters under the configuration $f=35$ and $c=80$, this would correspond to the optimal class separabilities $\Delta r_{\mathrm{PR}, \mathrm{opt}}=7.1 \mathrm{~dB}$ and $\Delta r_{\mathrm{TC}, \text { opt }}=6.0 \mathrm{~dB}$ in the model presented in Section IV-B1, which is relatively consistent with the expected values according to past studies.

The a priori probabilities of both classes are also derived from this GIS data, and they lead to $p(B)=0.75$.

The effects of SAR system parameters are simulated either by varying the classification threshold at different values (channel gain imbalance or radiometric stability) or by explicitly degrading the images (ambiguity). The corresponding probability of classification error is calculated based on the GIS data in order to model the impact of these SAR parameters at different values. 



Fig. 13. Effect of parameter $d$ on (left) the overall classification accuracy and on (right) the additional error for the PR method.
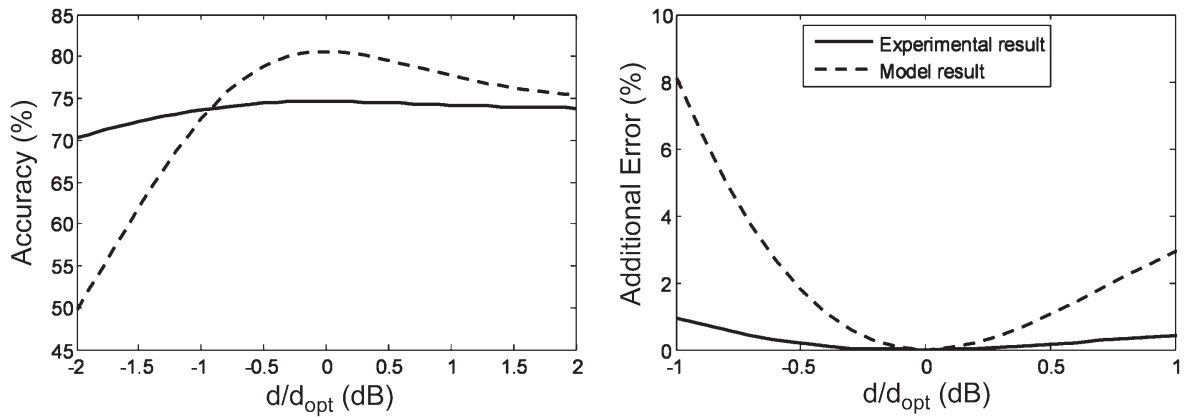

Fig. 14. Effect of parameter $d$ on (left) the overall classification accuracy and on (right) the additional error for the TC method.

The effects of crosstalk cannot be addressed here as the HV channel is not available.

\section{A. Sensitivity to Parameter d}

In this section, we intend to test the sensitivity of the classification methods to parameter $d=r_{t} / r_{0}$, where $r_{t}$ is the retained classification threshold and $r_{0}=\sqrt{r_{A} r_{B}}$. As shown in Sections III-A and B, parameter $d$ represents the channel gain imbalance for the PR method and the radiometric stability for the TC method. Because of potential inaccuracies in the class parameter estimations $r_{A}$ and $r_{B}$, it seems that the optimal classification threshold $r_{\mathrm{opt}}$ can be retrieved with more accuracy than $r_{0}$ by testing a wide range of values for $r_{t}$ and by identifying the value that minimizes the error. Therefore, it seems more relevant to consider the distance $d^{\prime}=r_{t} / r_{\mathrm{opt}}$ rather than $d$. This parameter can be calculated from $d$ theoretically: $d^{\prime}=d / d_{\mathrm{opt}}$, where $d_{\mathrm{opt}}$ is given in (12), which depends on $\Delta r, L$, and $p(B)$.

The methods are applied on the PRI images after calibration, georeferencing, and spatial filtering to reduce the effect of speckle. The initial number of looks in the georeferenced data is calculated to be $L_{i}=1.8$, which is consistent with the nominal number of looks given for that product (one in range and two in azimuth). In this area, the fields are relatively large, with $F \approx 200 \mathrm{~m}$, and the pixel size is $R=12.5 \mathrm{~m}$. This allows a $7 \times 7$ window to be used in the low-pass box filter. The resulting number of looks is $L_{e}=34.3$, which is in agreement with (24), indicating that $22.0<L_{e}<44.1$.
The experimental results with the real data and the theoretical outputs from the error model are compared in Fig. 13 for the PR method and in Fig. 14 for the TC method. In both figures, the overall classification accuracy is plotted in the left-hand side, and the additional error due to $d$ is shown in the right-hand side, both as a function of $d / d_{\text {opt }}$.

For the PR method, the experimental accuracy is lower than the theoretical accuracy calculated by the model (around 12\%). This can be partly explained by the fact that the GIS database used to assess the experimental accuracy is partially inaccurate. In fact, a visual inspection reveals that the differences between the rice map obtained from the SAR images and from the GIS are spatially localized rather than randomly distributed, indicating that the GIS may not be up to date. The additional errors are, however, in very good agreement, with an absolute difference lower than $0.6 \%$. For the TC method, the classification accuracies calculated in the two approaches compare rather badly. The additional errors have similar trends but with different amplitudes, with the modeled values being around three times as big as the experimental values. These discrepancies can however be explained by the high value of the satellite repeat cycle, which makes the classification accuracy highly dependent on the timing of the acquisitions. The calculated value of $\Delta r_{\mathrm{TC}}$ is, therefore, not necessarily representative of the $\Delta r_{90 \%}$ parameter, and the assessment of $\Delta r_{\mathrm{TC}, \text { opt }}$ may be incorrect.

Based on the results obtained in the PR method, the model can be effectively used to assess the effects of channel gain imbalance or radiometric stability. 

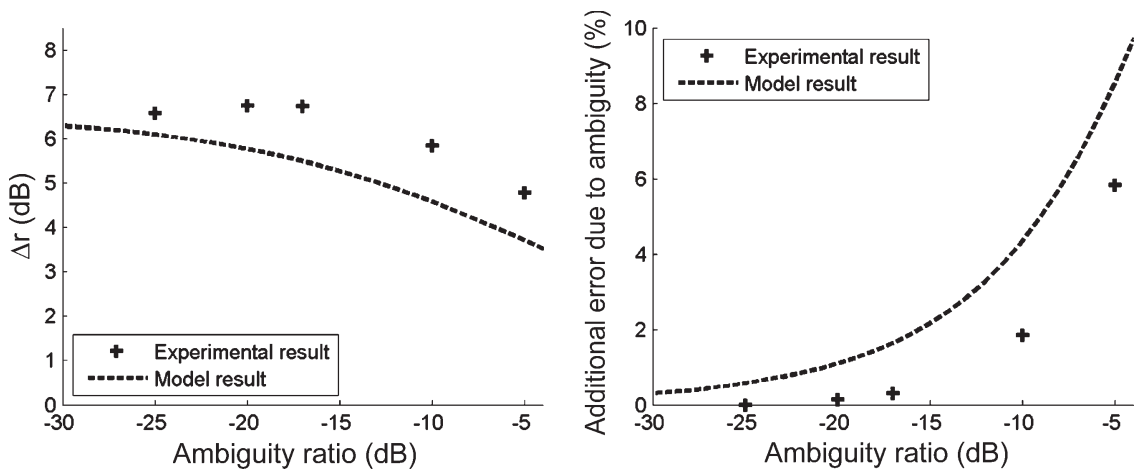

Fig. 15. (Left) Effect of the ambiguity ratio on the class separability and (right) additional error due to ambiguity for the PR method.

\section{B. Sensitivity to the Ambiguity Ratio}

The ambiguity is simulated by degrading the SLC images according to the relationship given in (20) for each polarization and each date and for five ambiguity ratio values: $-5,-10$, $-17,-20$, and $-25 \mathrm{~dB}$. The -5 and $-10 \mathrm{~dB}$ values are not realistic but are nevertheless simulated to test the sensitivity of the model. Contrary to the analysis in Section IV-A, where the source of the ambiguity is set at a constant backscatter value of $0 \mathrm{~dB}$ to simulate the worst possible case, a real scene is selected here from another subset of the image. This is therefore expected to produce lower additional errors than the theoretical study.

After simulating the ambiguity in the complex amplitude images in slant-range geometry, the backscattering coefficient is computed, a $3 \times 15$ low-pass box filter is applied to reduce the speckle while taking into account the different pixel spacing in range and azimuth, and the images are georeferenced to the GIS geometry using tie points. The number of looks of the georeferenced images is calculated to be $L=19$.

Fig. 15 shows the variations of the $\Delta r$ parameter and of the additional error due to ambiguity as a function of the ambiguity ratio, calculated for the five experimental values and simulated by the error model for the PR method. The error model is run with $p(B)=0.75, L=19, \Delta r=6.57 \mathrm{~dB}$, and $I_{1, B}=-6 \mathrm{~dB}$, which is calculated from the $\mathrm{HH}$ and VV images.

As predicted by the model, the class separability decreases in the experimental data set when the ambiguity ratio increases, but its decrease is expectedly less important in the experimental data than in the model. Consequently, the experimental additional error increases with ambiguity, which is similar to the theoretical error, but to a lower extent. The observed experimental trends related to the error are well described by the model.

\section{CONClusion}

A new expression for the probability of error in classification methods based on a SAR intensity ratio has been provided, introducing a supplementary parameter corresponding to a bias between measured and true ratios.

This error model has been used to assess the impact of SAR system parameters on the classification performance for the two-class problem. The effect of channel gain imbalance and radiometric stability has been directly estimated in the general case. When the two classes are fairly separable (i.e., the difference between their mean ratios is higher than $4 \mathrm{~dB}$ ), it was found that the typical values of the channel gain imbalance lead to a negligible additional error, while the impact of radiometric instability can be significant in its upper range for some values of the equivalent number of looks in classifications based on a TC. The case of close classes discriminated through the use of a high equivalent number of looks is very sensitive to channel gain imbalance or to radiometric stability for the methods based, respectively, on a $\mathrm{PR}$ or a $\mathrm{TC}$, and it requires low values of these calibration parameters. The effect of crosstalk on the backscattering coefficient was modeled and found to be critical when cross-polarizations are involved, unless stringent requirements are met. Degradation due to ambiguity was found to be negligible when the ambiguity ratio is lower than $-30 \mathrm{~dB}$. In other cases, some configurations can lead to critical additional errors and should be avoided (low class separability and low number of looks). Typical temporal backscattering profiles have been modeled to investigate the impact of observation frequency in both methods. Simulations have demonstrated the importance of the temporal sampling, corresponding to the time-lapse between two consecutive satellite's repeatpass orbits, for methods based on a TC. This reinforces the expectations set on the coming high-repetition SAR mission (Sentinel-1).

The model has been validated by comparing its outputs to experimental results obtained from real SAR data used in rice field mapping methods. The sensitivity of the error model to channel gain imbalance, radiometric stability, and ambiguity is tested, and it shows a relatively good agreement between experimental and theoretical trends.

In summary, the error model proposed in this paper is expected to provide a useful tool for SAR mission design, which is also suitable for the development of classification methods based on existing instruments.

\section{APPENDIX I}

The retained classification threshold is $r_{t}=d \cdot r_{0}$

$P E_{B}=\int_{0}^{r_{t}} p\left(r \mid r_{B}\right) d r=\frac{\Gamma(2 L)}{\Gamma(L)^{2}} \int_{0}^{d \cdot r_{0}} \frac{r_{B}^{L} r^{L-1}}{\left(r_{B}+r\right)^{2 L}} d r=\frac{\Gamma(2 L)}{\Gamma(L)^{2}} I_{B}$. 
The substitution $s=r_{B}+r$ and the use of the generalized binomial theorem lead to

$$
\begin{aligned}
I_{B} & =\int_{r_{B}}^{d \cdot r_{0}+r_{B}} \frac{r_{B}^{L}\left(s-r_{B}\right)^{L-1}}{s^{2 L}} d s \\
& =r_{B}^{L} \cdot \int_{r_{B}}^{d \cdot r_{0}+r_{B}} \frac{\sum_{k=0}^{\infty}\left(\begin{array}{c}
L-1 \\
k
\end{array}\right)(-1)^{k} r_{B}^{k} s^{L-1-k}}{s^{2 L}} d s .
\end{aligned}
$$

The series $\quad S_{n}=\sum_{k=0}^{n}\left(\begin{array}{c}L-1 \\ k\end{array}\right)\left((-1)^{k} r_{B}^{k} s^{L-1-k} / s^{2 L}\right)=$ $\sum_{k=0}^{n} u_{k}(s)$ converges normally on the interval

$$
\begin{aligned}
D & =\left[r_{B}, d \cdot r_{0}+r_{B}\right] \text { to } \sum_{k=0}^{\infty}\left\|u_{k}\right\|_{\infty}=\sum_{k=0}^{\infty} \sup _{D}\left(\left|u_{k}(s)\right|\right) \\
& =\sum_{k=0}^{\infty}\left|u_{k}\left(r_{B}\right)\right|=\frac{\left(r_{B}+r_{B}\right)^{L-1}}{r_{B}^{2 L}} .
\end{aligned}
$$

$S_{n}$ is therefore uniformly convergent, which allows the interchange of the series and integral signs

$$
\begin{aligned}
I_{B} & =\sum_{k=0}^{\infty}\left(\begin{array}{c}
L-1 \\
k
\end{array}\right) \int_{r_{B}}^{d \cdot r_{0}+r_{B}} \frac{(-1)^{k} r_{B}^{L+k}}{s^{L+k+1}} d s \\
& =\sum_{k=0}^{\infty}\left(\begin{array}{c}
L-1 \\
k
\end{array}\right)\left[\frac{(-1)^{k+1} r_{B}^{L+k}}{(L+k) s^{L+k}}\right]_{r_{B}}^{d \cdot r_{0}+r_{B}} \\
& =\sum_{k=0}^{\infty}\left(\begin{array}{c}
L-1 \\
k
\end{array}\right) \frac{(-1)^{k}}{(L+k)}\left[1-\left(\frac{r_{B}}{d \cdot r_{0}+r_{B}}\right)^{L+k}\right] \\
& =\sum_{k=0}^{\infty}\left(\begin{array}{c}
L-1 \\
k
\end{array}\right) \frac{(-1)^{k}}{(L+k)}\left[1-\left(\frac{\sqrt{\Delta r}}{d+\sqrt{\Delta r}}\right)^{L+k}\right] .
\end{aligned}
$$

Similarly, we have

$$
P E_{A}=\int_{r_{t}}^{\infty} p\left(r \mid r_{A}\right) d r=\frac{\Gamma(2 L)}{\Gamma(L)^{2}} \int_{d \cdot r_{0}}^{\infty} \frac{r_{A}^{L} r^{L-1}}{\left(r_{A}+r\right)^{2 L}} d r=\frac{\Gamma(2 L)}{\Gamma(L)^{2}} I_{A}
$$

and it can be found, with the substitution $s=r_{A}+r$, that

$$
I_{A}=\sum_{k=0}^{\infty}\left(\begin{array}{c}
L-1 \\
k
\end{array}\right) \frac{(-1)^{k}}{(L+k)}\left(\frac{1}{1+d \sqrt{\Delta r}}\right)^{L+k}
$$

leading to $P E=p(A) \cdot h_{L}\left(d^{2} \cdot \Delta r\right)+p(B) \cdot\left(h_{L}(0)-\right.$ $\left.h_{L}\left(d^{2} / \Delta r\right)\right)$ with

$$
h_{L}(\Delta r)=\frac{\Gamma(2 L)}{\Gamma(L)^{2}} \sum_{k=0}^{\infty}\left(\begin{array}{c}
L-1 \\
k
\end{array}\right) \frac{(-1)^{k}}{(L+k)}\left(\frac{1}{1+\sqrt{\Delta r}}\right)^{L+k} .
$$

It can be noticed that

$$
h_{L}(\Delta r)+h_{L}\left(\frac{1}{\Delta r}\right)=\left|P E_{A}+h_{L}(0)-P E_{B}\right|_{d=1} .
$$

By substituting the variable $s=r_{0}^{2} / r$, we find that

$$
\begin{aligned}
\left|P E_{A}\right|_{d=1} & =\int_{r_{0}}^{\infty} p\left(r \mid r_{A}\right) d r=\frac{\Gamma(2 L)}{\Gamma(L)^{2}} \int_{r_{0}}^{\infty} \frac{r_{A}^{L} r^{L-1}}{\left(r_{A}+r\right)^{2 L}} d r \\
& =\frac{\Gamma(2 L)}{\Gamma(L)^{2}} \int_{r_{0}}^{0} \frac{r_{A}^{L}}{r_{B}^{L}} \cdot \frac{r_{B}^{L}\left(r_{0}^{2} / s\right)^{L-1}}{\left(r_{A}+r_{0}^{2} / s\right)^{2 L}} \cdot \frac{-r_{0}^{2}}{s^{2}} d s \\
& =\frac{\Gamma(2 L)}{\Gamma(L)^{2}} \int_{0}^{r_{0}} \frac{r_{B}^{L} s^{L-1}}{\left(r_{B}+s\right)^{2 L}} d s=\left|P E_{B}\right|_{d=1} .
\end{aligned}
$$

Noticing that $h_{L}(0)=\left|P E_{A}\right|_{d=0}=\int_{0}^{\infty} p\left(r \mid r_{A}\right) d r=1$ leads to $h_{L}(\Delta r)+h_{L}(1 / \Delta r)=1$ and, finally, equation (9).

\section{APPENDIX II}

Let us assume that $n$ classes are considered, with class $i$ characterized by a mean ratio $r_{i}$, and $r_{1}<r_{2}<\cdots<r_{n}$. The distance between two consecutive mean ratios is $\Delta r_{i}=$ $r_{i+1}-r_{i}$, with $i$ varying from $i$ to $n-1$, and the corresponding retained classification thresholds are $r_{t i}=\sqrt{r_{i} r_{i+1}}$.

Considering that all classes are equiprobable $(p(i)=1 / n)$, the overall probability of error for $n>2$ is

$$
\begin{aligned}
& P E=\frac{1}{n}\left[\int_{r_{t 1}}^{\infty} p\left(r \mid r_{1}\right) d r\right. \\
&+\sum_{i=2}^{n-1}\left(\int_{r_{t i}}^{\infty} p\left(r \mid r_{i}\right) d r+\int_{0}^{r_{t(i-1)}} p\left(r \mid r_{i}\right) d r\right) \\
&\left.+\int_{0}^{r_{t(n-1)}} p\left(r \mid r_{n}\right) d r\right] \\
&=\frac{1}{n}\left[\sum_{i=1}^{n-1}\left(\int_{r_{t i}}^{\infty} p\left(r \mid r_{i}\right) d r+\int_{0}^{r_{t i}} p\left(r \mid r_{i+1}\right) d r\right)\right] .
\end{aligned}
$$

Equation (5) gives the probability of error of the twoclass problem for classes $i$ and $i+1$ with equal a priori probabilities

$$
P E\left(\Delta r_{i}, L\right)=\frac{1}{2}\left(\int_{r_{t i}}^{\infty} p\left(r \mid r_{i}\right) d r+\int_{0}^{r_{t i}} p\left(r \mid r_{i+1}\right) d r\right)
$$

which leads to equation (13)

$$
P E=\frac{2}{n} \sum_{i=1}^{n-1} P E\left(\Delta r_{i}, L\right)=\frac{2(n-1)}{n} \operatorname{mean}_{i}\left[P E\left(\Delta r_{i}, L\right)\right] .
$$




\section{ACKNOWLEDGMENT}

The authors would like to thank Prof. S. Quegan for his valuable suggestions on this manuscript as part of the review of A. Bouvet's Ph.D. thesis.

\section{REFERENCES}

[1] S. R. Cloude and E. Pottier, "An entropy based classification scheme for land applications of polarimetric SAR," IEEE Trans. Geosci. Remote Sens., vol. 35, no. 1, pp. 68-78, Jan. 1997.

[2] A. Freeman and S. L. Durden, "A three-component scattering model for polarimetric SAR data," IEEE Trans. Geosci. Remote Sens., vol. 36, no. 3, pp. 963-973, May 1998.

[3] L. Ferro-Famil, E. Pottier, and J.-S. Lee, "Unsupervised classification of multifrequency and fully polarimetric SAR images based on the H/A/Alpha-Wishart classifier," IEEE Trans. Geosci. Remote Sens., vol. 39, no. 11, pp. 2332-2342, Nov. 2001.

[4] J.-S. Lee, M. R. Grunes, T. L. Ainsworth, L.-J. Du, D. L. Schuler, and S. R. Cloude, "Unsupervised classification using polarimetric decomposition and the complex Wishart classifier," IEEE Trans. Geosci. Remote Sens., vol. 37, no. 5, pp. 2249-2258, Sep. 1999.

[5] J.-S. Lee, M. R. Grunes, E. Pottier, and L. Ferro-Famil, "Unsupervised terrain classification preserving polarimetric scattering characteristics," IEEE Trans. Geosci. Remote Sens., vol. 42, no. 4, pp. 722-731, Apr. 2004.

[6] E. Rignot and J. van Zyl, "Change detection techniques for ERS-1 SAR data," IEEE Trans. Geosci. Remote Sens., vol. 31, no. 4, pp. 896-906, Jul. 1993.

[7] S. Takeuchi, T. Konishi, Y. Suga, and S. Kishi, "Comparative study for flood detection using JERS-1 SAR and Landsat TM data," in Proc. IGARSS, 1999, pp. 873-875.

[8] R. Andreoli and H. Yesou, "Assessment of the change detection procedure dedicated to flood monitoring using ENVISAT wide-swath mode data," in Proc. Dragon Symp., 2008, [CD-ROM].

[9] F. Ribbes, T. Le Toan, J. Bruniquel, N. Floury, N. Stussi, S. C. Liew, and U. R. Wasrin, "Deforestation monitoring in tropical regions using multitemporal ERS/JERS SAR and InSAR data," in Proc. IGARSS, 1997, pp. $1560-1562$.

[10] R. Hadria, B. Duchemin, F. Baup, T. Le Toan, A. Bouvet, G. Dedieu, and M. Le Page, "Combined use of optical and radar satellite data for the detection of tillage and irrigation operations: Case study in Central Morocco," Agric. Water Manage,, vol. 96, pp. 1120-1127, 2009.

[11] T. Le Toan, F. Ribbes, L.-F. Wang, N. Floury, K.-H. Ding, J. A. Kong, M. Fujita, and T. Kurosu, "Rice crop mapping and monitoring using ERS-1 data based on experiment and modelling results," IEEE Trans. Geosci. Remote Sens., vol. 35, no. 1, pp. 41-56, Jan. 1997.

[12] F. Ribbes and T. Le Toan, "Rice field mapping and monitoring with RADARSAT data," Int. J. Remote Sens., vol. 20, no. 4, pp. 745-765, Mar. 1999.

[13] V. Malinovsky, S. Sandven, A. Mironov, and A. Korinenko, "Identification of oil spills based on ratio of alternating polarization images from ENVISAT," in Proc. IGARSS, 2007, pp. 1326-1329.

[14] P. Ferrazzoli, L. Guerriero, and G. Schiavon, "Experimental and model investigation on radar classification capability," IEEE Trans. Geosci. Remote Sens., vol. 37, no. 2, pp. 960-968, Mar. 1999.

[15] J. Paris, "Radar backscattering properties of corn and soybeans at frequencies of 1.6, 4.75 and $13.3 \mathrm{GHz}$," IEEE Trans. Geosci. Remote Sens., vol. GRS-21, no. 3, pp. 392-400, Jul. 1983.

[16] A. Bouvet, T. Le Toan, and N. Lam Dao, "Monitoring of the rice cropping system in the mekong delta using ENVISAT/ASAR dual polarization data," IEEE Trans. Geosci. Remote Sens., vol. 47, no. 2, pp. 517-526, Feb. 2009

[17] G. Satalino, F. Mattia, T. Le Toan, and M. Rinaldi, "Wheat crop mapping by using ASAR AP data," IEEE Trans. Geosci. Remote Sens., vol. 47, no. 2, pp. 527-530, Feb. 2009.

[18] B. Scheuchl, D. Flett, R. Caves, and I. Cumming, "Potential of RADARSAT-2 data for operational sea ice monitoring," Can. J. Remote Sens., vol. 30, pp. 448-461, 2004.

[19] R. Touzi, A. Lopes, and P. Bousquet, "A statistical and geometrical edge detector for SAR images," IEEE Trans. Geosci. Remote Sens., vol. 26, no. 6, pp. 764-773, Nov. 1988.

[20] G. Moser and S. B. Serpico, "Generalized minimum-error thresholding for unsupervised change detection from SAR amplitude imagery,"
IEEE Trans. Geosci. Remote Sens., vol. 44, no. 10, pp. 2972-2982, Oct. 2006.

[21] S. Quegan, T. Le Toan, J. J. Yu, F. Ribbes, and N. Floury, "Multitemporal ERS SAR analysis applied to forest monitoring," IEEE Trans. Geosci. Remote Sens., vol. 38, no. 2, pp. 741-753, Mar. 2000.

[22] W. F. Kibble, "A two-variate gamma type distribution," Sankhya, Indian J. Stat., vol. 5, no. 2, pp. 137-150, 1941.

[23] E. Xekalaki, J. Panaretos, and S. Psarakis, "A predictive model evaluation and selection approach-The correlated gamma ratio distribution," in Stochastic Musings: Perspectives from the Pioneers of the Late 20th Century, J. Panaretos, Ed. Mahwah, NJ: Lawrence Erlbaum, 2003, pp. $188-202$.

[24] A. Freeman, "A new system model for radar polarimeters," IEEE Trans. Geosci. Remote Sens., vol. 29, no. 5, pp. 761-767, Sep. 1991.

[25] CEOS, SAR data products format standards, 1989.

[26] J. Bruniquel, "Contribution de données multi-temporelles à l'amélioration radiométrique et à l'utilisation d'images de radars à synthèse d'ouverture," Ph.D. dissertation, Univ. Paul Sabatier Toulouse, Toulouse, France, 1996.

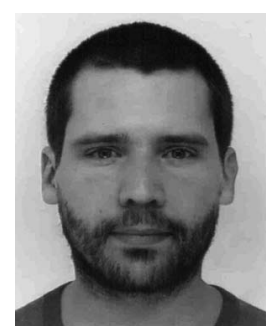

Alexandre Bouvet (M'09) received the Engineering degree from the Ecole Nationale Supérieure d'Aéronautique et de l'Espace, Toulouse, France, in 2004 and the Ph.D. degree from Université Paul Sabatier-Toulouse III, Toulouse, in the Centre d'Etudes Spatiales de la Biosphère, in 2009.

Since 2010, he has been a Postdoctoral Fellow with the European Commission Joint Research Center, Ispra, Italy, where he is working on the use of SAR data for the analysis of tropical forest cover dynamics. His interests include SAR image processing and the use of remote sensing data to monitor vegetated areas for environmental applications.

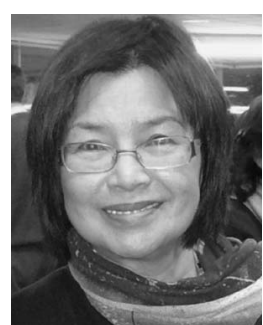

Thuy Le Toan received the Ph.D. degree in atomic and nuclear physics from the University of Toulouse, Toulouse, France.

She has been the Head of the Remote Sensing Research Team, Centre d'Etudes Spatiales de la Biosphère, Toulouse. She has been a Project Coordinator of European experimental campaigns and the Principal Investigator (PI) of several ERS, JERS, SIR-C/XSAR, RADARSAT, ENVISAT, and ALOS-PALSAR satellite projects. She has also been involved in numerous studies for the E.U., ESA, NASA, JAXA, and national organizations on the use of SAR in monitoring land surfaces. She has been the Leader of the BIOMASS satellite mission candidate of the 2005 ESA Call for Ideas for the Next Earth Explorer Core Mission. The mission was retained for the prefeasibility phase, in May 2006, and for the phase A in March 2009. At present, she is the Cochair of the ESA Phase-A Earth Explorer BIOMASS Mission Advisory Group and the European-China Dragon project "The role of cropland and grassland in the carbon budget of China," the PI of the Planet Action project "The impact of climate and human activities on the environment of the Mekong delta," and a member of the JAXA Kyoto and Carbon Initiatives on the use of ALOS data for deforestation monitoring and biomass measurement, with impacts on the forest carbon budget. Her research activity has been in the area of remote sensing for land applications, including the experimentation and modeling of microwave interaction with agricultural and forested media. Her current interest is on the use of Earth Observation to characterize the land processes and their changes under the effects of climate and human activities and to quantify their impacts on the carbon cycle. 


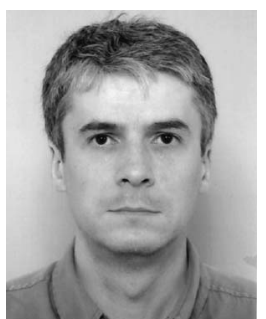

Nicolas Floury (M'07) received the Diplôme d'Ingénieur from Ecole Nationale Supérieure des Télécommunications, Paris, France, in 1993 and the Ph.D. degree from Université Paris 7-Denis Diderot, Paris, in 1999.

Since 1999, he has been with the ESTEC Facility, European Space Agency, Noordwijk, The Netherlands, where he is currently heading the Wave Interaction and Propagation Section. The section contributes to research and development in the fields of electromagnetic (optical to microwave) propagation through and interaction with natural media (atmosphere, land, and sea surface) and supports on these aspects the agency's programs in the domains of satellite telecommunication, navigation, and Earth observation. His technical interests include the signal processing and electromagnetic modeling applied to microwave interaction with natural media, and he has published on the applications of synthetic aperture radars and of microwave radiometers to remote sensing.

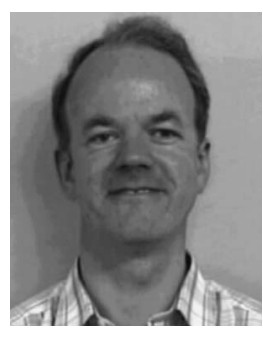

Trevor Macklin was born in Belfast, U.K., in 1956. He received the B.Sc. degree in physics from Queen's University, Belfast, in 1977. He continued his postgraduate work on extragalactic radio sources at the Mullard Radio Astronomy Observatory, Cambridge University, Cambridge, U.K., and received the Ph.D. degree in 1982.

$\mathrm{He}$ is currently with the Remote Sensing Team, BAE SYSTEMS Advanced Technology Centre, Chelmsford Essex, U.K., where his main research interests are in the applications and image quality of synthetic aperture radar.

Dr. Macklin is a Fellow of the Royal Astonomical Society. 